\title{
A Discrete Dislocation Analysis of Hydrogen-Assisted Mode-I Fracture
}

\author{
N. Irani ${ }^{\mathrm{a}}$, J.J.C. Remmers ${ }^{\mathrm{a}}$, V.S. Deshpande ${ }^{\mathrm{a}, \mathrm{b}, *}$ \\ ${ }^{a}$ Department of Mechanical Engineering, Eindhoven University of Technology, P.O. Box 513, 5600 MB Eindhoven, The \\ Netherlands. \\ ${ }^{b}$ Department of Engineering, Cambridge University, Trumpington Street, Cambridge CB2 1PZ, UK.
}

\begin{abstract}
Fracture of engineering alloys in the presence of hydrogen commonly occurs by decohesion along grain boundaries via a mechanism known as hydrogen induced decohesion (HID). This mechanism is investigated here by analysing the mode-I fracture of a single crystal with plastic flow in the crystal described by discrete dislocation plasticity (DDP) and material separation (decohesion) modelled using a cohesive zone formulation. The motion of dislocations is assumed to be unaffected by hydrogen diffusion. While the cohesive strength is assumed to be reduced proportional to the local hydrogen concentration. Two limiting cases are analysed: (i) the fast diffusion limit where the hydrogen within the material is assumed to be at chemical equilibrium throughout the loading so that there is a high hydrogen concentration in the regions of high hydrostatic stress around dislocations and near the crack tip and (ii) the slow diffusion limit where we assume that there is no appreciable hydrogen diffusion over the duration of loading and thus the hydrogen concentration remains spatially uniform as in a stress-free material. The lower cohesive strength at high hydrogen concentrations results in reduced dislocation activity around the crack tip and a reduction in the material toughness. In fact, at the highest hydrogen concentrations analysed here, crack growth primarily occurs in an elastic manner. However, surprisingly the calculations predicted that the toughness in the fast diffusion case was no more than $12 \%$ lower compared to the slow diffusion case suggesting that the stress concentrations due to the dislocation structures and the crack tip fields have only a minor effect on the toughness reduction in the presence of hydrogen. The DDP calculations are finally used to investigate the sensitivity of the material toughness to the grain boundary cohesive strength. The calculations show that the toughness of materials with a small cohesive opening at the peak cohesive traction are more sensitive to hydrogen loading. We speculate that this result might be used as a guide in grain boundary engineering to design alloys that are less sensitive to hydrogen embrittlement by the HID mechanism.
\end{abstract}

Keywords: Hydrogen embrittlement, Stressed-assisted diffusion, Discrete dislocation plasticity

\section{Introduction}

A large number of engineering alloys (steel, nickel, etc.) when exposed to hydrogen, suffer from a loss of ductility and toughness, which may lead to sudden, premature failure. Consequently, the adverse effects of hydrogen embrittlement must be included in engineering design for applications such as pipelines and nuclear power plants that come into contact with water, hydrocarbons or hydrogen gas. Hydrogen embrittlement is also critical for welded joints since hydrogen take-up can arise from the use of damp electrodes in electric welding operations.

The mechanisms of hydrogen embrittlement remain controversial with a range of mechanisms proposed in an attempt to explain observations; see recent review by Robertson et al. (2015). Of the variety of these mechanisms two dominate the literature, viz. Hydrogen Induced Decohesion (HID) and Hydrogen Enhanced Localised Plasticity (HELP). According to the HID mechanism, hydrogen that has accumulated at a crack

${ }^{*}$ Corresponding author E-mail address: vsd@eng.cam.ac.uk 
tip reduces the cohesive strength giving rise to a reduced fracture toughness (Oriani, 1970; Troiano, 1960). In contrast, the HELP mechanism (Birnbaum and Sofronis, 1994) assumes that hydrogen redistribution occurs around dislocations and it reduces the elastic interaction energy between dislocations and the associated Peierls stress. Gangloff (2003) argued that HID is the dominant mechanism in high strength alloys on the basis that a wide range of micromechanical fracture toughness models of HID are able to predict (i) the threshold stress intensity factor $K_{T H}$ and (ii) the crack growth rate $d a / d t$ versus $K$ response for hydrogen exposed alloys. Serebrinsky et al. (2004) support the view of Gangloff (2003) by developing a quantitative HID based model: they assumed that a cohesive zone exists at a crack tip and the strength of the cohesive zone drops with increasing local hydrogen concentration. By suitable adjustments of material parameters, this model was able to predict the observed incubation time for crack initiation, the effect of hydrogen concentration upon $K_{T H}$ and the effect of temperature upon $d a / d t$. Jiang and Carter (2004) also supported the HID mechanism; they obtained the dependence of surface energy upon hydrogen concentration via a first principles calculation. Recently, Novak et al. (2010) proposed a synergetic effect of the HID and HELP mechanisms. They argued that the HELP mechanism reduces the length of pile-ups on carbides situated at the grain boundaries. Simultaneously, the presence of hydrogen reduces the cohesive toughness of the grain boundaries (HID), giving rise to premature inter-granular fracture.

There is growing evidence from atomistic calculations that hydrogen reduces the cohesive strength $\Sigma_{\max }$ and toughness of grain boundaries, see for example Geng et al. (2005). This fact is also supported by experimental evidence that grain boundary engineering significantly reduces the susceptibility of nickel to hydrogen embrittlement (Bechtlea et al., 2013). The effect of cohesive strength on the toughness of metals was investigated in a pioneering study by Tvergaard and Hutchinson (1992). They employed continuum J2-flow theory of plasticity to model plastic deformation in the metal with uniaxial tensile yield strength $\sigma_{Y}$ and a cohesive surface to model the separation process. Their calculations demonstrated that the normalised mode-I steady-state toughness $K_{\mathrm{ss}} / K_{0}\left(\Sigma_{\max }\right)$ increased with increasing $\Sigma_{\max } / \sigma_{Y}$, where $K_{\mathrm{ss}}$ is the steadystate toughness and $K_{0}\left(\Sigma_{\max }\right)$ is the toughness of the corresponding elastic solid with cohesive strength $\Sigma_{\max }$. In fact, for an ideally plastic solid under plane strain conditions the toughness was unbounded as $\Sigma_{\max } / \sigma_{Y} \rightarrow 3$. While this model of Tvergaard and Hutchinson (1992) qualitatively explains the reason for the reduction in the toughness of the metal due to the presence of hydrogen (i.e. hydrogen reduces the cohesive strength which in turn reduces toughness), it has some key drawbacks: (i) different metals have varying degrees of susceptibility to hydrogen but the model of Tvergaard and Hutchinson (1992) suggests that there exists a unique $K_{\mathrm{ss}} / K_{0}$ versus $\Sigma_{\max } / \Sigma_{Y}$ relation; (ii) the prediction that the toughness is unbounded as $\Sigma_{\max } / \sigma_{Y} \rightarrow 3$ is unrealistic, as the cohesive strength of grain boundaries is expected to be significantly greater than 3 times the uniaxial tensile yield strength of the metal, $\sigma_{Y}$ and (iii) most HID models require stresses far in excess of the yield strength to be generated at the crack tip in order to draw in sufficient levels of hydrogen to the crack and trigger HID.

Some of these drawbacks have been addressed by employing the overall framework of Tvergaard and Hutchinson (1992), but rather than using J2-flow theory of plasticity, investigators have employed enhanced plasticity theories such as strain gradient plasticity (Chen et al., 1999) and discrete dislocation plasticity (Cleveringa et al., 2000) to model the plastic deformation of metals during fracture. These theories are capable of modelling the size dependent yield strength of metals and thus predict a finite fracture toughness for $\Sigma_{\max } / \sigma_{Y} \gg 3$ where again $\sigma_{Y}$ is the corresponding uniaxial tensile yield strength. More recently, Martínez-Pañeda et al. (2016) have used strain gradient plasticity theories to analyse HID in terms of the Gerberich (2012) model. However, the material length scale used in their model was a fitting parameter chosen to give the required high stresses at the crack tip: it is unclear whether the large length scale $(5 \mu \mathrm{m})$ chosen by Martínez-Pañeda et al. (2016) is realistic. In discrete dislocation plasticity the dislocation structures that develop and the associated length scales are a natural outcome of the solution to the boundary value problem and here we aim to clarify the Hydrogen Induced Decohesion (HID) mechanism using this methodology.

The outline of the paper is as follows: First, we briefly describe the discrete dislocation plasticity (DDP) framework to analyse crack growth and the model used to include the effect of hydrogen on the decohesion process. Second, computational results are presented for the effects of hydrogen diffusion rate, grain boundary cohesive strength and hydrogen concentration on the toughness of the material. Motivated by 
these results we conclude by presenting a map that summarises the effect of hydrogen on the toughness as a function of two material non-dimensional groups.

\section{Formulation of the problem}

\subsection{Discrete dislocation plasticity formulation for Mode-I crack growth}

Consider a specimen under remote tensile loading as sketched in Fig. 1(a). We envisage hydrogen induced decohesion (HID) resulting in fracture along grain boundaries as shown in Fig. 1(a) with the crack growing primarily in mode- $\mathrm{I}^{1}$. Given the mode-I nature of crack growth we consider the idealised situation of an infinitely long crack in a two-dimensional single crystal subjected to far field mode-I loading as sketched in Fig. 1(b). Symmetry about the crack plane representing a grain boundary is assumed so that we need to consider only half of the crystal. This small-scale yielding problem is analysed with plasticity assumed to be confined to a rectangular window of $L_{p} \times h_{p}$ inside of which dislocations are treated discretely; see Fig. 1(b). The calculations are terminated before dislocations reach the boundary of this window. Remote from the crack tip, displacements corresponding to the isotropic, linear elastic mode-I singular fields which are characterised by the mode-I stress intensity factor $K_{I}$ are applied. Furthermore, crack initiation and growth are modelled using a cohesive surface (Deshpande et al., 2002); the properties of this cohesive surface will be specified later. The boundary value problem formulation and the numerical implementation follow that in Cleveringa et al. (2000) and Deshpande et al. (2002) where further details and additional references are given.

At each time step, an increment of the mode-I stress intensity factor $\dot{K}_{I} \Delta t$ is prescribed, where $\dot{K}_{I}$ is the applied rate of $K_{I}$ and $\Delta t$ is the time increment used in the numerical calculations. At the current instant, the stress and strain states of the body is known, so that the forces acting on all dislocations can be calculated. On the basis of these forces we update the dislocation structure, which involves the motion of dislocations, the generation of new dislocations, their mutual annihilation, their pinning at and releasing from obstacles, and their exit into the open crack. After this, the increments in the stress and strain fields are solved from the incremental version of the virtual work equation

$$
\int_{V} \sigma_{i j} \delta \varepsilon_{i j} \mathrm{~d} V-\frac{1}{2} \int_{S_{\mathrm{coh}}} t_{i} \delta \Delta_{i} \mathrm{~d} S=\int_{S_{\mathrm{ext}}} t_{i} \delta u_{i} \mathrm{~d} S .
$$

Here, $V$ is the volume of the region analysed, $S_{\text {ext }}$ is the external surface and $S_{\text {coh }}$ is the surface where cohesive tractions operate. Furthermore, $\sigma_{i j}$ are the components of the stress tensor, $t_{i}$ are the cohesive traction components, $u_{i}$ are the displacement field components, $\Delta_{i}$ are the components of the displacement jump across the cohesive surface, and

$$
\begin{aligned}
& \varepsilon_{i j}=\frac{1}{2}\left(u_{i, j}+u_{j, i}\right), \\
& t_{i}=\sigma_{i j} n_{j}
\end{aligned}
$$

where $n_{i}$ are the components of the unit outward normal on $S_{\text {coh }}$ or $S_{\text {ext }}$. Superposition is used to determine the velocity, strain and stress rate fields in the body with the new dislocation distribution (Van der Giessen and Needleman, 1995) such that

$$
\dot{u}_{i}=\dot{\tilde{u}}_{i}+\dot{\hat{u}}_{i}, \quad \dot{\varepsilon}_{i j}=\dot{\tilde{\varepsilon}}_{i j}+\dot{\hat{\varepsilon}}_{i j}, \quad \dot{\sigma}_{i j}=\dot{\tilde{\sigma}}_{i j}+\dot{\hat{\sigma}}_{i j}
$$

The $\left(^{\sim}\right)$ fields are the sum of the fields of the individual dislocations, in their current configuration that give rise to tractions $\tilde{t}_{i}$ and displacements $\tilde{u}_{i}$ on the boundary of the body. The individual dislocation fields are

\footnotetext{
${ }^{1}$ While we have motivated the problem in terms of intergranular fracture, we emphasise here that our analysis is valid for any weak interface within the material.
} 
those for an edge dislocation in a traction-free half-space, with the traction-free surface corresponding to the crack plane $x_{2}=0$. The $\left(^{\wedge}\right)$ fields represent the image fields that correct for the actual boundary conditions. Contrary to the $\left(^{\sim}\right)$ fields, the latter are non-singular and are obtained with a finite element method.

At each time-step, the stress analysis consists of three main computational stages: (i) determining the Peach-Koehler forces on the dislocations using the current stress state; (ii) determining the changes in the dislocation structure caused by the motion of dislocations, the generation of new dislocations, their mutual annihilation, their possible pinning at and releasing from obstacles; and (iii) determining the stress and strain state for the updated dislocation arrangement.

Knowing the current stress state, the Peach-Koehler force $f^{(I)}$ acting on the $I$-th dislocation is determined by

$$
f^{(I)}=n_{i}^{(I)}\left(\hat{\sigma}_{i j}+\tilde{\Sigma}_{i j}^{(I)}+\sum_{J \neq I} \sigma_{i j}^{(J)}\right) b_{j}^{(I)}
$$

with $n_{i}^{(I)}$ the slip plane normal and $b_{j}^{(I)}$ the Burgers vector of dislocation $I$ while $\tilde{\Sigma}_{i j}^{(I)}$ is the non-singular component of $\tilde{\sigma}_{i j}^{(I)}$ related to the traction-free surface of the half-plane solution employed here. The direction of this force is along the slip plane and normal to the dislocation line. The Peach-Koehler force includes the long-range interactions with all other dislocations in the material. This force determines the evolution of the dislocation structure, accounting for glide, generation, annihilation, pinning at and releasing from obstacles.
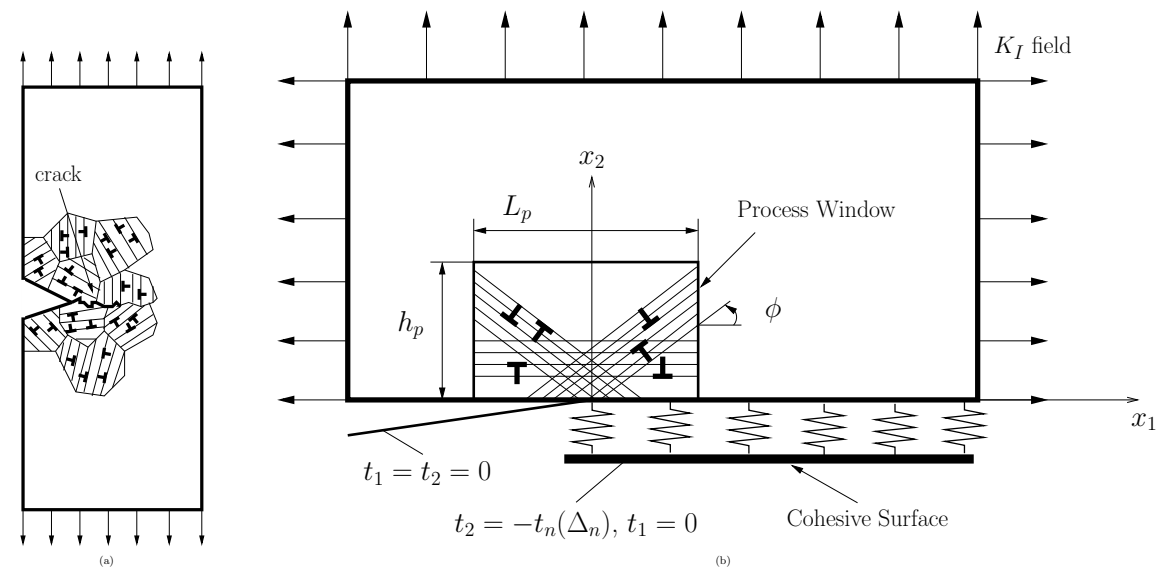

Figure 1: (a) Sketch of tensile loading of a polycrystalline alloy with decohesion occurring along grain boundaries and the crack growing primarily in mode-I. (b) Sketch of the idealised mode-I boundary value problem analysed for crack growth in a single crystal.

When inertia is neglected, the magnitude of the glide velocity $v^{(I)}$ of dislocation $I$ can be obtained using the drag relation below,

$$
v^{(I)}=\frac{1}{B} f^{(I)}
$$

where $B$ is the drag coefficient.

New dislocation pairs are generated by simulating Frank-Read sources. This mechanism consists of bowing of a dislocation pinned at two points, and the subsequent instability of the bowed dislocation. This results in the formation of a dislocation loop and preserving the original pinned dislocation. Here, every Frank-Read segment is represented by a point source on a slip plane, and two nucleated edge dislocations of opposite signs represent the cross section of the newly generated loop. This mechanism is approximated as 
follows: If the resolved shear stress acting on the source exceeds a critical value $\tau_{\text {nuc }}$, for a sufficiently long time $t_{\text {nuc }}$, two opposite edge dislocations are nucleated. The distance $L_{\text {nuc }}$ between the dislocations is equal to

$$
L_{\mathrm{nuc}}=\frac{E}{4 \pi\left(1-\nu^{2}\right)} \frac{b}{\tau_{\text {nuc }}},
$$

where $E$ is the Young's modulus and $\nu$ is the Poisson's ratio. Annihilation of two dislocations with opposite Burgers vector occurs when they are sufficiently close together. This is modelled by eliminating two dislocations when they are within a critical annihilation distance $L_{\mathrm{e}}$.

Finally, obstacles to dislocation motion are modelled as fixed points on a slip plane. A dislocation gliding on a slip plane can be stopped and pinned by an obstacle at the same slip plane. Pinned dislocations can only pass the obstacles when their Peach-Koehler force exceeds an obstacle dependent value $\tau_{\text {obs }} b$, where $\tau_{\text {obs }}$ is the obstacle strength. We emphasise here that we will assume that the dislocation dynamics (i.e. dislocation motion, nucleation, annihilation etc.) is unaffected by the presence of hydrogen. This is consistent with the fact that we neglect the HELP mechanism in this model and only investigate the effect of HID. This assumption is also in line with the fact that the tensile response prior to fracture is typically unaffected by hydrogen (Gangloff, 2003) suggesting that hydrogen does not affect the dislocation dynamics.

\subsection{Oriani's theory for hydrogen distribution}

We now summarise the Oriani (1970) theory for the relative distribution of hydrogen in the lattice and at traps. Hydrogen in a metal is either stored at normal interstitial lattice sites (NILS) or is trapped at micro-structural defects such as dislocations, grain boundaries, interfaces and carbides. The concentration of hydrogen at NILS, also called the lattice hydrogen concentration, is given by $C_{L}=\theta_{L} \beta N_{L}$ where $\theta_{L}$ is the fraction of occupied interstitial lattice sites, $\beta$ is the number of NILS per lattice atom and $N_{L}$ is the number of lattice atoms per unit volume. Similarly, the concentration of hydrogen trapped at any given site is $C_{T}=\theta_{T} \alpha N_{T}$ where $\theta_{T}$ is the fraction of occupied trapping sites, $\alpha$ is the number of atom sites per trap and $N_{T}$ is the number of traps per unit volume. Oriani's equation states the equilibrium value of occupancy ratio of sites, in terms of an equilibrium constant $K^{2}$ as

$$
\frac{\theta_{T}}{1-\theta_{T}}=K \frac{\theta_{L}}{1-\theta_{L}}
$$

In turn, $K$ is related to the trap binding energy $\Delta H$, the gas constant $R$ and the absolute temperature $T$ according to

$$
K=\exp \left(-\frac{\Delta H}{R T}\right)
$$

Since $\theta_{L} \ll 1$, Eq. (7) can be re-phrased as

$$
\theta_{T} \approx \frac{K \theta_{L}}{1+K \theta_{L}}=\frac{C_{L} /\left(\beta N_{L}\right)}{C_{L} /\left(\beta N_{L}\right)+\exp (\Delta H / R T)}
$$

Typically, for a given metal $\beta$ and $N_{L}$ are well-known from the lattice structure; see for example Sofronis and McMeeking (1989). However, there is greater uncertainty about the value of $\Delta H$ for the different types of traps. For example, Novak et al. (2010) suggest that $\Delta H$ takes values in the range from $-15 \mathrm{~kJ} / \mathrm{mol}$ to $-80 \mathrm{~kJ} / \mathrm{mol}$ depending on whether the traps are dislocations, grain boundaries or carbide particles. Nevertheless, given $\Delta H$ for grain boundaries and the lattice hydrogen concentration in the vicinity of a grain boundary one can use Eq. (9) to predict the fraction of available sites in the grain boundary occupied by hydrogen atoms (i.e. $\left.\theta_{T}\right)$.

\footnotetext{
${ }^{2}$ This equilibrium is established on the time-scale of femto to nano-seconds set by the frequency of lattice vibrations and trap energies. It is thus independent of the lattice diffusivity.
} 


\subsubsection{The lattice hydrogen concentration}

To predict the hydrogen coverage of grain boundaries (i.e. $\theta_{T}$ ) we require to estimate the lattice hydrogen concentration $C_{L}$ in the vicinity of grain boundaries. Recall that the lattice hydrogen is free to diffuse in the material and is driven by the chemical potential gradients. Here, we shall consider two limiting situations: (i) a fast diffusion limit (or equivalently a slow loading rate limit) where the hydrogen can diffuse within the lattice fast enough so that it can be assumed in chemical equilibrium at every instant of loading and (ii) a slow diffusion limit (or equivalently a fast loading rate limit) where there is no time for diffusion of the lattice hydrogen over the duration of the test. In all cases, the trapping kinetics are assumed to be fast enough so that the trapped hydrogen is always in local equilibrium with the lattice hydrogen and therefore may be obtained by Oriani's theory described above.

We consider a specimen in hydrogen atmosphere (e.g. acidic or alkaline bath) such that there is ample supply of hydrogen to feed the specimen as needed. The specimen is first immersed in this bath prior to loading and then is allowed to attain an equilibrium state. At this point the lattice and trapping sites within the specimen fill up with hydrogen such that the trapped hydrogen is in equilibrium with the lattice hydrogen. Furthermore, the lattice hydrogen with concentration $C_{L}^{0}$ is spatially uniform within the specimen with chemical potential

$$
\mu=\mu_{0}+R T \ln \left(C_{L}^{0}\right),
$$

where $\mu_{0}$ is the chemical potential in the standard state and $C_{L}^{0}$ is given by the equality $\mu=\mu_{R}$, where $\mu_{R}$ is the chemical potential of hydrogen in the atmosphere within which the specimen is immersed. Thus, in experiments $C_{L}^{0}$ is typically adjusted by changing the hydrogen atmosphere in which the experiment is conducted.

We first consider the fast diffusion limit. If loading is applied to the specimen, the local hydrostatic stress $\sigma_{h}\left(x_{i}\right)=\sigma_{k k}\left(x_{i}\right) / 3$ at location $x_{i}$ in the specimen alters the chemical potential of the lattice hydrogen to

$$
\mu\left(x_{i}\right)=\mu_{0}+R T \ln \left(C_{L}\left(x_{i}\right)\right)-\sigma_{h}\left(x_{i}\right) V_{\mathrm{H}},
$$

where $V_{\mathrm{H}}$ is the partial molar volume of hydrogen in solid solution. In the fast diffusion limit, since there is ample time for diffusion to occur within the specimen, $\mu\left(x_{i}\right)$ will be spatially uniform. Now recall that remote from the crack tip (or local stress concentrations where failure occurs) $\sigma_{h} \approx 0$ with $C_{L}\left(x_{i}\right)=C_{L}^{0}$. Equating the chemical potentials of point $x_{i}$ to that far field from the crack tip, we obtain

$$
C_{L}\left(x_{i}\right)=C_{L}^{0} \exp \left(\frac{\sigma_{h}\left(x_{i}\right) V_{\mathrm{H}}}{R T}\right) .
$$

On the other hand, in the slow diffusion limit there is no time for diffusion to occur over the duration of the test and we take $C_{L}\left(x_{i}\right)=C_{L}^{0}$. This implicitly assumes that negligible hydrogen is trapped within the dislocation fields as confirmed by the calculations presented in Appendix A. These expressions for the lattice hydrogen concentration can be substituted into Eq. (9) to obtain the coverage $\theta_{T}$ of a grain boundary at location $x_{i}$.

\subsection{Decohesion of grain boundaries}

Mode-I grain boundary decohesion is modelled by a cohesive surface with a normal traction separation relation given by

$$
t_{\mathrm{n}}\left(\Delta_{\mathrm{n}}\right)=\sigma_{\max } \frac{\Delta_{\mathrm{n}}}{\delta_{\mathrm{n}}} \exp \left(-\frac{\Delta_{\mathrm{n}}}{\delta_{\mathrm{n}}}+1\right),
$$

where $t_{n}$ is the normal cohesive traction when the normal cohesive separation is $\Delta_{n}$. The cohesive traction attains a maximum value of $\sigma_{\max }$ when the separation $\Delta_{n}=\delta_{n}$. Moreover, the cohesive work of separation 
follows as $\phi_{n}=e \sigma_{\max } \delta_{n}$, where $e$ is the Euler's number. In our work, we have assumed that our system fails due to HID mechanism and hence the work of separation gets affected by hydrogen concentration at grain boundaries. A fit to the first principles calculations of Jiang and Carter (2004) for aluminium suggests a relation of the form

$$
\frac{\phi_{n}\left(\theta_{T}\right)}{\phi_{n}\left(\theta_{T}=0\right)}=1-0.67 \theta_{T},
$$

while the calculations of Van der Ven and Ceder (2003) suggest that $\delta_{n}$ is insensitive to $\theta_{T}$. It then follows that hydrogen coverage primarily reduces the cohesive strength such that

$$
\frac{\sigma_{\max }\left(\theta_{T}\right)}{\Sigma_{\max }}=1-0.67 \theta_{T},
$$

where $\Sigma_{\max } \equiv \sigma_{\max }\left(\theta_{T}=0\right)$ is the strength of the grain boundary in the absence of hydrogen.

\subsection{Crack growth calculations}

In our calculations, we model the simplified problem of a single grain boundary which is loaded in mode-I as shown in Fig. 1(b). Along the $x_{2}=0$ surface we have boundary conditions

$$
t_{1}\left(x_{1}, 0\right)=t_{2}\left(x_{1}, 0\right)=0 \quad x_{1}<0,
$$

and

$$
t_{1}\left(x_{1}, 0\right)=0, t_{2}\left(x_{1}, 0\right)=-t_{n} \quad x_{1} \geq 0
$$

Furthermore, assumed symmetry about $x_{2}=0$ implies that the cohesive opening $\Delta_{n}$ is related to the displacement via the relation $\Delta_{\mathrm{n}}=2 u_{2}\left(x_{1}, 0\right)$.

Recall that the cohesive properties depend on $\theta_{T}$ which in turn is influenced by $C_{L}\left(x_{i}\right)$. Throughout this study, we specify hydrogen loading by a dimensionless parameter $\theta_{0}$ defined as

$$
\theta_{0}=\frac{C_{L}^{0} /\left(\beta N_{L}\right)}{C_{L}^{0} /\left(\beta N_{L}\right)+\exp (\Delta H / R T)} .
$$

The dependence of $\theta_{0}$ on $C_{L}^{0}$ at $T=300 K$ for $\mathrm{Al}$ with a density $2700 \mathrm{~kg} / \mathrm{m}^{3}$ is shown in Fig. 2 for $\Delta H$ values in the range of $-10 \mathrm{~kJ} / \mathrm{mol}$ to $-50 \mathrm{~kJ} / \mathrm{mol}$ (representative of the range of relatively shallow dislocation traps to deep grain boundary or carbide particle traps) with $\beta=6$ and $N_{L}=6.03 \times 10^{28} \mathrm{~m}^{-3}$. Over the typical practical values of $C_{L}^{0}$ ranging from 0.5 wppm to 5 wppm, shallow traps with $\Delta H=-10 \mathrm{~kJ} / \mathrm{mol}$ are almost depleted of hydrogen while deeper traps with $\Delta H=-50 \mathrm{~kJ} / \mathrm{mol}$ become saturated at very low values of $C_{L}^{0}$. However, for an intermediate trap binding energy of $\Delta H=-30 \mathrm{~kJ} / \mathrm{mol} \theta_{0}$ is sensitive to $C_{L}^{0}$ over the practical range of lattice hydrogen concentrations. In the literature, there is a considerable uncertainty on the values of $\Delta H$ for the various types of hydrogen traps. Here, we do not attempt to dwell into this issue and hence will present our results only in terms of $\theta_{0}$.

It now remains to relate the hydrogen concentration parametrised by $\theta_{0}$ to the cohesive properties. Given $\theta_{0}$ for the slow diffusion case, the cohesive properties are directly specified with $\theta_{T}=\theta_{0}$ and the relations in Section 2.3. However, in the fast diffusion case not only do the cohesive properties change with time but they are also spatially varying. In particular $\theta_{T}$ at time $t$ and location $x_{i}$ is given as

$$
\theta_{T}\left(x_{i}\right)=\frac{\theta_{0} \exp \left(\sigma_{h}\left(x_{i}\right) V_{\mathrm{H}} / R T\right)}{\theta_{0} \exp \left(\sigma_{h}\left(x_{i}\right) V_{\mathrm{H}} / R T\right)+\left(1-\theta_{0}\right)},
$$

and local cohesive strength follows from Eq. (15). In order to ensure that these relations are satisfied, we solve the boundary value problem summarised in Eq. (1) via an iterative solution as the appropriate gradients of the cohesive tractions required in the Newton method to solve Eq. (1) are difficult to estimate. 


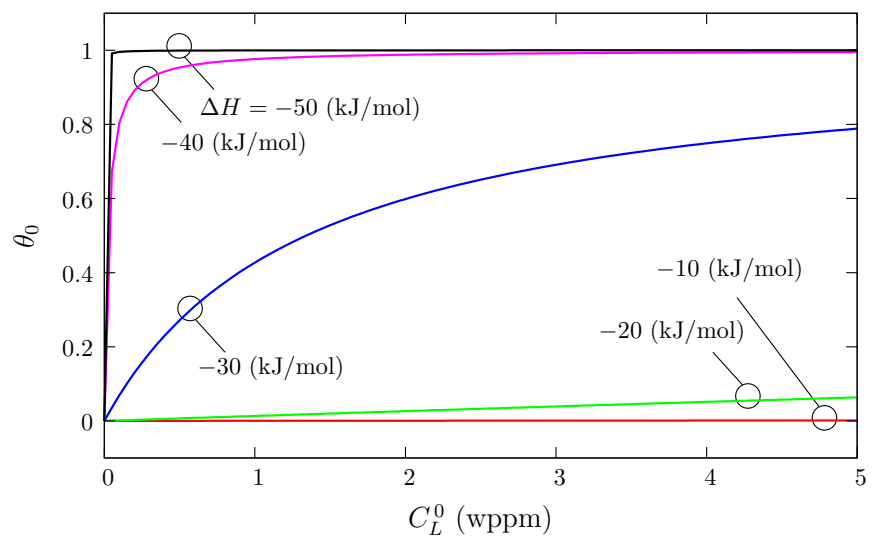

Figure 2: The sensitivity of hydrogen loading as parametrised by $\theta_{0}$ to the lattice hydrogen concentration $C_{L}^{0}$ for five selected values of trap binding energies $\Delta H$.

\subsection{Material and loading parameters}

The size of the region analysed (Fig. 1(b)) is $1000 \mu \mathrm{m} \times 500 \mu \mathrm{m}$ with a process window which is taken to have dimensions $15 \mu \mathrm{m} \times 15 \mu \mathrm{m}$. In all calculations, a finite element mesh of $250 \times 225$ bilinear quadrilateral elements was employed. Inside the process region, there is a graded $200 \times 200$ element mesh, with the minimum mesh spacing equal to $7.5 \mathrm{~nm}$ for the calculations with a cohesive property $\Sigma_{\max }=0.5 \mathrm{GPa}$. In the calculations with higher cohesive strength, the minimum mesh spacing is reduced to $5.3 \mathrm{~nm}$ and $4.2 \mathrm{~nm}$ for the $\Sigma_{\max }=0.75 \mathrm{GPa}$ and $\Sigma_{\max }=1.0 \mathrm{GPa}$ simulations, respectively. Resolving the dislocation dynamics requires a small time step of $\Delta t=0.5 \mathrm{~ns}$. Thus, the calculations were carried out with a rather high loading rate of $\dot{K}_{I}=100 \mathrm{GPa} \sqrt{\mathrm{m}} / \mathrm{s}$ in order to reduce the time required for the computations. The effect of loading rate is not explored here. However, Cleveringa et al. (2001) reported that varying the loading rate by two orders of magnitude did not change the crack growth behaviour qualitatively, although, of course, a strong tendency was found for increased plastic deformation at lower loading rates.

The process window of the crystal has three slip systems. Two of them are symmetrically oriented at $+60^{\circ}$ and $-60^{\circ}$ with respect to the crack plane $\left(x_{2}=0\right)$ and the third is parallel to the crack plane. For each slip system, the planes are equally spaced over the process window, with a spacing of $86 b$. Here, the Burgers vector $b$, for all dislocations was considered to be equal to $0.25 \mathrm{~nm}$. Initially, these slip planes are assumed not to have any mobile dislocations, but to have a random distribution of dislocation sources and obstacles, with a density equal to $25 \mu \mathrm{m}^{-2}$ and $39 \mu \mathrm{m}^{-2}$, respectively.

The calculations are reported for a temperature $T=300 \mathrm{~K}$ and the partial molar volume of hydrogen in a solid solution $V_{\mathrm{H}}$ is taken to be $2.0 \times 10^{-6} \mathrm{~m}^{3} / \mathrm{mol}$. The value of the drag coefficient is taken as $B=10^{-4} \mathrm{~Pa} \mathrm{~s}$, which is a representative value for aluminium (Kubin et al., 1992). The strength of the dislocation sources is randomly chosen from a Gaussian distribution with a mean strength $\bar{\tau}_{\text {nuc }}=50 \mathrm{MPa}$ and a standard deviation $0.2 \bar{\tau}_{\text {nuc }}$. The mean nucleation distance is $L_{\text {nuc }}=125 \mathrm{~b}$. The nucleation time for all sources is taken as $t_{\text {nuc }}=0.01 \mu \mathrm{s}$. All obstacles are taken to have the same strength $\tau_{\text {obs }}=150 \mathrm{MPa}$. The annihilation distance is specified as $L_{\mathrm{e}}=6 b$ (Kubin et al., 1992). The elastic constants are taken as $E=70 \mathrm{GPa}$ and $\nu=0.33$ giving a shear modulus $G=26.3 \mathrm{GPa}$.

Unless otherwise specified, all calculations are reported for cohesive properties (in the absence of hydrogen) of $\Sigma_{\max }=0.5 \mathrm{GPa}$ and $\delta_{n}=3 b$. The calculations are reported for values of $\theta_{0}$ in the range $0 \leq \theta_{0} \leq 1$ and the cohesive properties change due to the presence of hydrogen as described above. This choice of parameters with $E=70 \mathrm{GPa}$ ensures that the cohesive lengths are less than the critical lengths reported in Bhandakkar et al. (2010) above which artificial crack tip shielding effects occur. Further, with $\delta_{n}=3 b$, premature crack opening due to the nucleation of a dislocation near the crack tip is automatically avoided thereby circumventing the instabilities reported in Chakravarthy and Curtin (2011).

Finally, we note that in order to rule out any possible effects caused by a particular realisation of 
dislocation sources and obstacles, we have performed each simulation using 5 different samples. These samples are statistically identical, but in these specimens the specific placement of sources and obstacles vary. We report results that are the average of 5 different realisations.

\subsection{Tensile response of crystal}

In discrete dislocation plasticity the plastic yield response is an outcome of the boundary value problem. This fact is contrary to the continuum plasticity computations where the yield stress is an input into the calculation. Thus, to set a baseline for the material employed in these calculations with the properties described above, we performed a uniaxial tension calculation on a single crystal. Notice that the crystal studied in this section is intact and there are no cracks present in this simulation. Two of the slip planes are oriented at $\phi=60^{\circ}$ and $-60^{\circ}$ with respect to the $x_{2}$-axis and the third one is parallel to the $x_{2}$-axis, as shown in the inset of Fig. 3. The specimen has dimensions $16 \mu \mathrm{m} \times 4 \mu \mathrm{m}$ and a nominal tensile strain rate of $\dot{U} / L=50 / \mathrm{s}$ was employed. The predicted uniaxial tensile response is included in Fig. 3 and shows that the material is approximately elastic-perfectly plastic with a yield strength $\sigma_{Y} \approx 55 \mathrm{MPa}$. These results are reasonably insensitive to the loading rate and the specimen size over a range $L \geq 16 \mu \mathrm{m}$ and $W \geq 4 \mu \mathrm{m}$. We emphasise that this uniaxial tensile response is unaffected by hydrogen concentration as hydrogen only affects the decohesion strength of the grain boundaries via the cohesive properties, i.e. we are modelling HID and neglecting any effects of HELP.

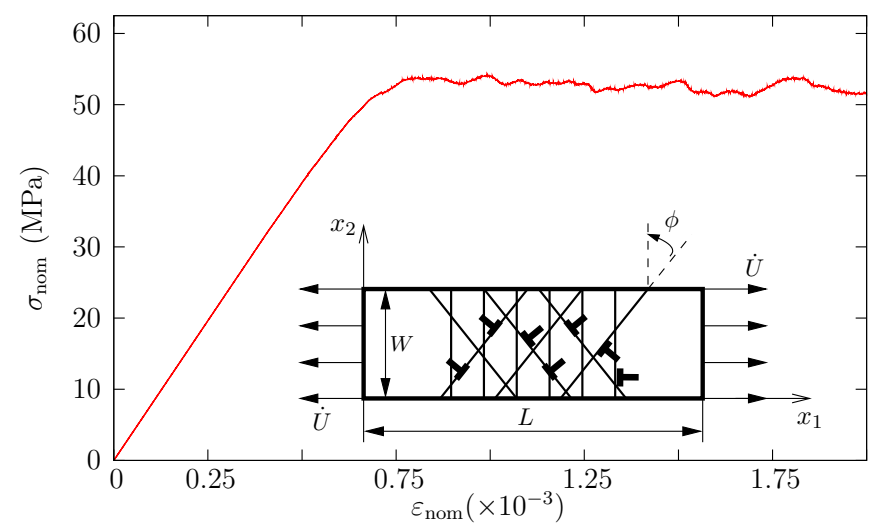

Figure 3: The uniaxial tensile nominal stress versus nominal strain response of the single crystal employed to obtain the yield properties. The inset shows the slip systems orientations.

\section{Numerical results}

We proceed to present DDP predictions for crack growth in the presence of hydrogen using the formulation outlined above. The results are primarily presented in terms of R-curves comprising the applied remote mode-I stress intensity factor $K_{I}$ versus the crack growth $\Delta a$. The crack tip location $x_{\text {tip }}$ is taken to be the point along the cohesive surface where the cohesive opening $\Delta_{n}=2 \delta_{n}$ and the crack growth $\Delta a=x_{\text {tip }}$ as the crack tip prior to loading is located at $x_{1}=0$. The applied $K_{I}$ is typically normalised by $K_{0}$ defined as

$$
K_{0}=\sqrt{\frac{E e \Sigma_{\max } \delta_{n}}{1-\nu^{2}}} .
$$

This value corresponds to the toughness of the corresponding elastic solid (i.e. fracture with no plasticity) in the absence of hydrogen loading. 


\subsection{Effect of hydrogen diffusion rate}

Predictions of R-curves for the material with reference cohesive properties (i.e. $\Sigma_{\max }=0.5 \mathrm{GPa}$ ) are included in Fig. 4(a) for both the fast and slow diffusion cases for four values of $\theta_{0}$ in the range $0 \leq \theta_{0} \leq 1$. The corresponding evolution of the dislocation density $\rho_{\text {dis }}$ (per unit area in the process window) with crack growth $\Delta a$ are included in Fig. 4(b). These predictions are averages over 5 simulations performed with different realisations of the source and obstacle distributions. We first review some general observations of the trends and then proceed to discuss the results in more detail.
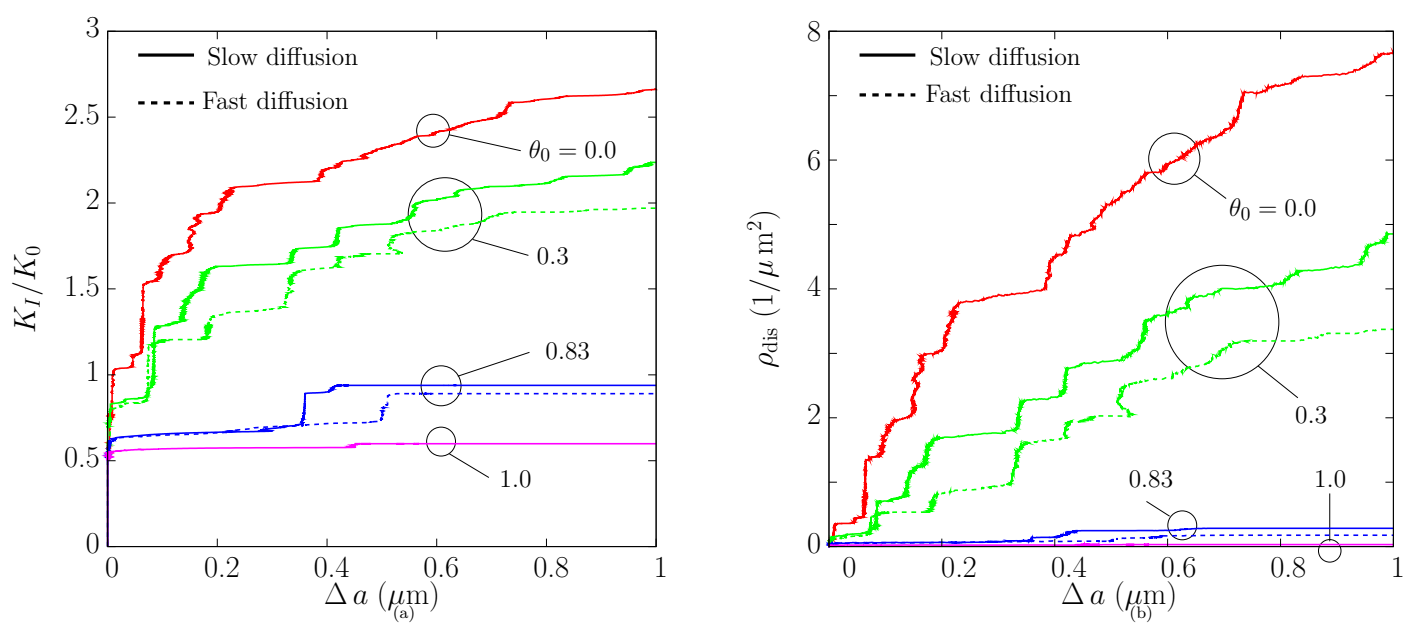

Figure 4: (a) Predictions of the R-curves in terms of normalised mode-I stress intensity factor $K_{I} / K_{0}$ versus crack extension $\Delta a$ and (b) the corresponding evolution of the dislocation density $\rho_{\text {dis }}$ versus $\Delta a$ for the slow and fast diffusion cases with the reference cohesive strength of $\Sigma_{\max }=0.5 \mathrm{GPa}$. The results are shown for four selected values of the hydrogen loading $\theta_{0}$.

In all cases, after the initiation of crack growth, the applied $K_{I}$ initially increases with increasing $\Delta a$ with the rate of increase $d K_{I} / d \Delta a$ reducing with increasing $\Delta a$, i.e. typical R-curve behaviour. Corresponding to the increase in $K_{I}$, the dislocation density $\rho_{\text {dis }}$ also increases with increasing $\Delta a$. Intriguingly, even for the $\theta_{0}=0$ case corresponding to no hydrogen loading, crack growth initiates at $K_{I} / K_{0} \approx 0.5$, i.e. in the presence of dislocation activity crack growth initiates at a lower $K_{I}$ value compared to a purely elastic material. This is rationalised by noting that dislocations play a dual role as discussed in Deshpande et al. (2002). On the one hand, dislocation motion results in plastic flow and dissipation resulting in an increasing R-curve but on the other hand dislocations act as stress concentrators that increase the stresses in their vicinity. These stress concentrations due to dislocations near the crack tip result in crack growth initiation at $K_{I} / K_{0}<1$. However, continued crack advance results in significant dislocation motion and consequent dissipation, resulting in the rising R-curve. By contrast, in an elastic material crack growth will initiate and grow unstably at $K_{I} / K_{0}=1$ as there is no dissipation mechanism present.

We first consider the case of slow diffusion. The results in Fig. 4(a) clearly show that with increasing $\theta_{0}$ there is negligible variation in the $K_{I}$ value for the initiation of crack growth. However, the R-curve effect is seen to reduce considerably with increasing $\theta_{0}$. In fact, at $\theta_{0}=1$ the material seems to behave in an approximately elastic manner with unstable crack growth occurring immediately after crack growth initiation. The corresponding $\rho_{\text {dis }}$ versus $\Delta a$ curves in Fig. 4(b) confirms this hypothesis: for a given $\Delta a$, the dislocation density is lower for the case with higher $\theta_{0}$. To further clarify the transition from a plastic to an elastic crack growth response we include in Fig. 5 contours of the crack tip opening stress $\sigma_{22}$ normalised by the cohesive strength $\Sigma_{\max }$ at the instant when the crack has grown by $\Delta a=1 \mu \mathrm{m}$ for $\theta_{0}=0,0.3$ and 0.83 . The dislocation structures at that instant of loading as well as the crack opening profile (with displacements $u_{2}$ magnified by a factor of 10) are also included in Fig. 5. Three key observations can be made from this 
figure:

(i) Consistent with Fig. 4(b) it is clear that the dislocation density decreases with increasing $\theta_{0}$.

(ii) The stress field in the $\theta_{0}=0$ case comprises distinct sectors that emanate from the crack tip along the slip directions which resembles the continuum plastic predictions of Rice (1987) for plastic fields in single crystals. By contrast in the $\theta_{0}=0.83$ case the stress field comprises lobes similar to the isotropic elastic stress fields.

(iii) The crack tip opening (i.e. blunting) decreases with increasing $\theta_{0}$.

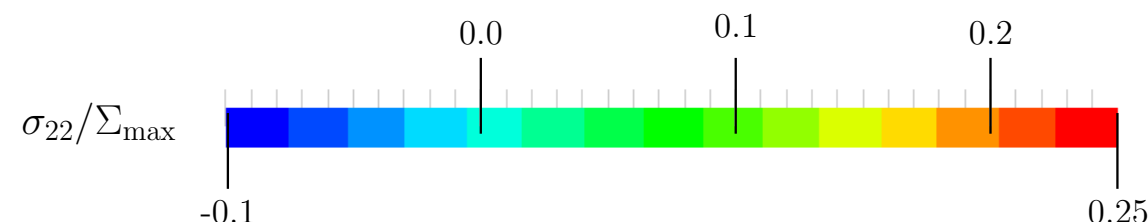

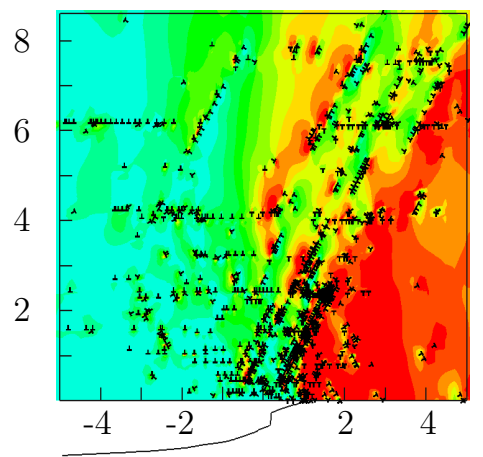

(a)

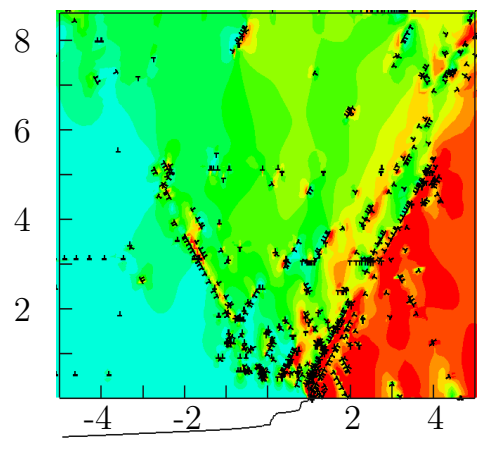

(b)

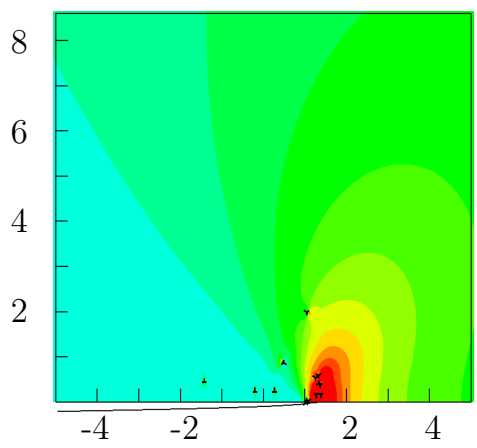

(c)

Figure 5: Predictions of the distribution of the normalised crack tip opening stress around the crack tip at $\Delta a=1 \mu \mathrm{m}$ for the slow diffusion case with the reference cohesive strength $\Sigma_{\max }=0.5 \mathrm{GPa}$. Results are shown for hydrogen loading parametrised by (a) $\theta_{0}=0$, (b) $\theta_{0}=0.3$ and (c) $\theta_{0}=0.83$. The corresponding dislocation structures and the crack tip profile with displacement $u_{2}$ magnified by a factor of 10 are also included in the figures. The dimensions on the $x_{1}$ - and $x_{2}$-axes are in $\mu \mathrm{m}$.

These observations all confirm that the reduction in the R-curve effect with increasing $\theta_{0}$ is due to the fact that crack growth occurs in a more elastic manner with increasing hydrogen loading. This phenomena can be understood by recalling that in the slow diffusion case the cohesive strength for a given $\theta_{0}$ is given directly from Eq. (15) with $\theta_{T}=\theta_{0}$, i.e. cohesive strength decreases linearly with increasing $\theta_{0}$. In the cohesive formulation, crack growth occurs approximately when the crack tip stress attains the peak cohesive traction at the opening $\Delta_{n}=\delta_{n}$ (after that point the cohesive relation is softening and continued cohesive opening typically occurs in an unstable manner). As the value of $\theta_{0}$ decreases we need to attain a higher peak traction $\sigma_{\max }$ in order for the crack to propagate. This requires a higher applied remote $K_{I}$ which in turn results in more dislocation activity near the crack tip and consequently higher plastic dissipation and a larger R-curve effect. This is similar to the continuum plasticity predictions of Tvergaard and Hutchinson (1992) with the caveat that continuum plasticity would predict an unbounded toughness if $\sigma_{\max } / \sigma_{\mathrm{Y}}>3$. However, in the DDP case here, even though $\sigma_{\max }=\Sigma_{\max }=0.5 \mathrm{GPa}$ when $\theta_{0}=0$ and $\sigma_{Y}=55 \mathrm{MPa}$ (i.e. $\left.\sigma_{\max } / \sigma_{\mathrm{Y}}=9.09\right)$ we predict a finite toughness.

In order to summarise these findings, we define a steady-state toughness $K_{\mathrm{ss}}$ and a corresponding steadystate dislocation density $\rho_{\mathrm{ss}}$ as the applied $K_{I}$ and dislocation density $\rho_{\text {dis }}$ at a crack extension $\Delta a=1 \mu \mathrm{m}$. These results for $K_{\mathrm{ss}}$ and $\rho_{\mathrm{ss}}$ are summarised in Figs. 6(a) and 6(b), respectively as a function of the hydrogen loading parametrised by $\theta_{0}$ (also see Appendix $\mathrm{C}$ for a discussion on the variability of the discrete 
dislocation predictions between the different realisations). Both $K_{\mathrm{ss}}$ and $\rho_{\mathrm{ss}}$ decrease with increasing $\theta_{0}$ for the reasons discussed above. This is in agreement with experimental observations (Gangloff, 2003) in that hydrogen loading results in a reduction in the fracture toughness of the material. In fact, for $\theta_{0}>0.8$ the dislocation density approaches zero consistent with the fact that crack growth occurs primarily in an elastic manner.

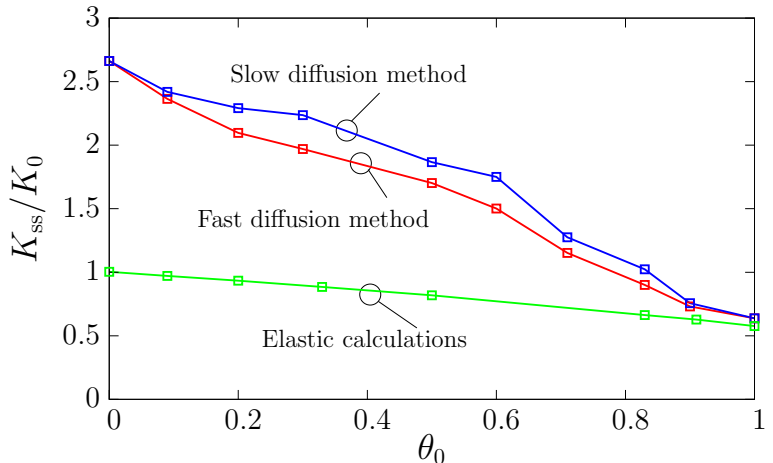

(a)

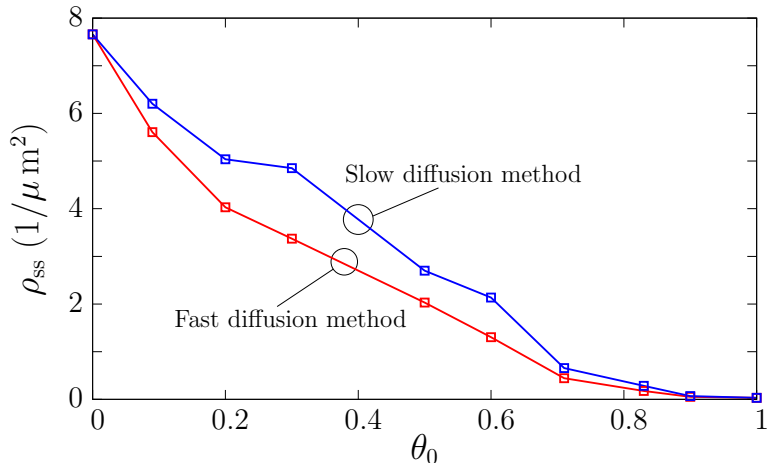

(b)

Figure 6: Predictions of the (a) normalised steady-state toughness $K_{\mathrm{ss}} / K_{0}$ and (b) steady-state dislocation density $\rho_{\mathrm{ss}}$ as a function of the hydrogen loading parametrised by $\theta_{0}$. Results are shown for both the slow and fast diffusion cases for the reference cohesive strength of $\Sigma_{\max }=0.5 \mathrm{GPa}$. In (a) also the predictions for the toughness of a purely elastic solid in the presence of hydrogen for the slow diffusion case are included.

In order to quantify the role of plasticity (or dislocation activity) in the hydrogen embrittlement process, it is worth comparing the DDP predictions of toughness in Fig. 6(a) with the toughness of a purely elastic solid in the presence of hydrogen for the slow diffusion case. Recall that elastic fracture occurs when $K_{I}=K_{0}$ with no R-curve effect and thus in the case of the elastic solid, $K_{\mathrm{ss}}$ directly follows from Eqs. (15) and (18) as,

$$
\frac{K_{\mathrm{ss}}\left(\theta_{0}\right)}{K_{0}}=\sqrt{1-0.67 \theta_{0}}
$$

This prediction is included in Fig. 6(a). It is clear that the reduction in toughness due to hydrogen loading is significantly larger when dislocation activity is present compared to the purely elastic case. This is due to the fact that in the absence of hydrogen, dislocation activity or plastic dissipation significantly enhances the toughness of the material. As hydrogen loading reduces the cohesive strength, material toughness decreases because of two reasons: (i) the inherent crack tip toughness $K_{0}\left(\theta_{0}\right)$ decreases due to the reduction in the cohesive strength and (ii) the plastic dissipation also reduces as crack opening requires a smaller stress and hence lower dislocation activity in the surrounding material. The first effect is present for both the plastic and purely elastic material but it is a relatively minor effect. The second effect is typically the significantly larger contributor to the toughness of a plastic material as discussed by Suo et al. (1993) and thus it is the reduced dislocation activity which is the primary contributor to the reduction in the toughness of a metal in the presence of hydrogen. Thus, dislocations do not directly affect the HID mechanism but rather have a large indirect effect in the sense that the reduction in dislocation activity due to the reduction in the cohesive strength because of hydrogen loading, is the primary cause for the observed toughness reductions.

We now consider the case of fast diffusion. Predictions of the R-curves and the corresponding $\rho_{\text {dis }}$ versus $\Delta a$ are included in Figs. 4(a) and 4(b), respectively for the fast diffusion case. The predictions for the fast diffusion case are very similar to the slow diffusion case with both the R-curve effects and $\rho_{\text {dis }}$ decreasing with increasing $\theta_{0}$, i.e. crack growth becomes more elastic with increasing $\theta_{0}$ as for the slow diffusion case. Differences between the two cases only occur at the intermediate value of $\theta_{0}=0.3$. At intermediate values 


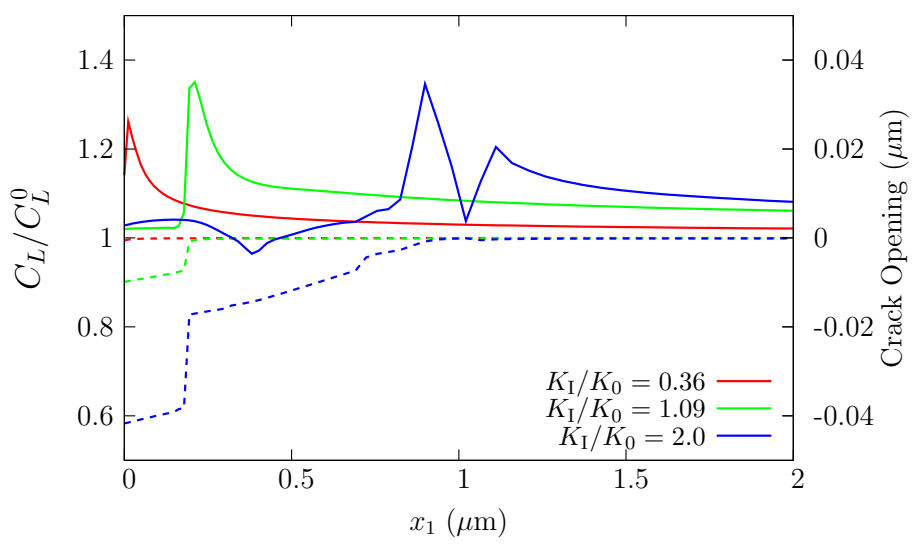

(a)
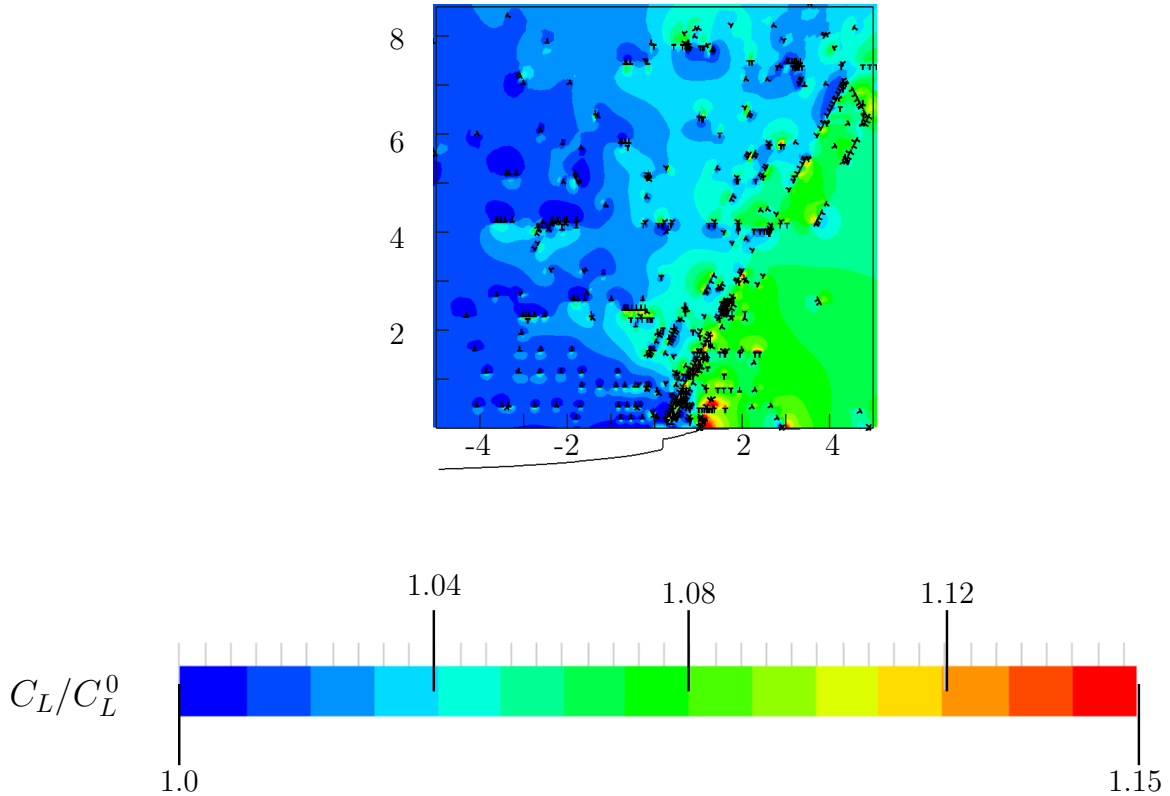

(b)

Figure 7: (a) The normalised hydrogen concentration $C_{L} / C_{L}^{0}$ along $x_{2}=0$ at three selected values of the normalised applied mode-I stress intensity factor $K_{I} / K_{0}$ for the fast diffusion case and the reference cohesive strength $\Sigma_{\max }=0.5 \mathrm{GPa}$ (solid lines). The crack tip profiles at each of the three values of $K_{I} / K_{0}$ are shown by dashed lines in (a). (b) The corresponding predictions of the distribution of normalised hydrogen concentration $C_{L} / C_{L}^{0}$ around the crack tip at $\Delta a=1 \mu \mathrm{m}$. The dislocation structure is included in (b) along with the crack tip profile with displacements $u_{2}$ magnified by a factor of 10 . The dimensions on the $x_{1}$ - and $x_{2}$-axes are in $\mu \mathrm{m}$. 
of $\theta_{0}$ the predicted toughness in the fast diffusion case is slightly lower compared to the slow diffusion case. This suggests that the dependence of the local hydrogen concentration (as parametrised by $\theta_{T}$ ) on the stress state even near the crack tip is mild.

In order to understand the source of the enhanced reduction in material toughness for the fast diffusion case we include in Fig. 7(a) predictions of the local hydrogen concentrations $C_{L} / C_{L}^{0}$ along $x_{2}=0$ at three selected values of applied $K_{I} / K_{0}$ for the $\theta_{0}=0.3$ fast diffusion case. Knowing the stress field (and hence $\sigma_{h}\left(x_{i}\right)$ ), the predictions for $C_{L} / C_{L}^{0}$ follow directly from Eq. (12). Also included in Fig. $7($ a) are the crack tip profiles (with displacements $u_{2}$ magnified by a factor of 10) in order to accurately show the location of the crack tip at the three loading instants considered here. It is clear that away from the crack tip the hydrogen concentration is typically enhanced by $10 \%$ or less compared to the value of $C_{L}^{0}$. However, in the very near vicinity of the crack tip the high local tensile hydrostatic stresses draw in more hydrogen with $C_{L} / C_{L}^{0} \approx 1.4$. This local increase in the hydrogen concentration results in a further reduction in the cohesive strength compared to the strength reduction due to the far field hydrogen concentration $C_{L}^{0}$ and therefore results in a further reduction in the toughness of material. This local increase in the hydrogen concentration is further exemplified in the contour plot of $C_{L} / C_{L}^{0}$ in the vicinity of the crack tip at $\Delta a=1 \mu \mathrm{m}$ in Fig. 7(b). It is clear that any significant increase in hydrogen concentration is very localised near the crack tip with only small increases in $C_{L}$ over $C_{L}^{0}$ in the remainder of the material. In fact, rather surprisingly, the plot in Fig. 7 (b) suggests that the hydrogen concentration is not significantly affected by the stress fields due to the dislocation structures around the crack tip. In Appendix A we further clarify this and show that over any realistic range of dislocation density the dislocation stress fields do not trap any significant amounts of hydrogen, re-enforcing the conclusion that hydrogen concentration is only enhanced as we asymptotically approach the crack tip. As a corollary, we conclude that stresses near crack tip can only build to sufficiently high levels to draw in significant levels of hydrogen if the material around the crack tip is "starved" of dislocations and thus remains elastic. This dislocation starvation phenomena is unlikely to occur around crack tips in metals and not covered by the range of parameters (i.e. source densities etc.) considered here.

The steady-state toughness and dislocation density results for the fast diffusion case are computed in a manner similar to the slow diffusion case and included in Figs. 6(a) and 6(b), respectively. At intermediate values of $\theta_{0}$ both $K_{\mathrm{ss}}$ and $\rho_{\mathrm{ss}}$ are reduced by a maximum of $12 \%$ and $30 \%$, respectively due to the local tensile hydrostatic stress fields drawing in more hydrogen as discussed above. However, this effect is surprisingly rather small with the main trends being adequately captured by the slow diffusion limit. Recall that in the slow diffusion case hydrogen concentration is assumed to remain fixed at $C_{L}^{0}$ throughout the body at all times and the reduction in the cohesive strength due to local hydrostatic tensile stresses drawing in more hydrogen is not included. We thus conclude that changes in the hydrogen concentrations due to local dislocation and crack tip stress fields play a relatively minor role. Therefore, for the remainder of this study we restrict our analysis to the slow diffusion limit.

\subsection{Effect of grain boundary cohesive strength}

The calculations in the previous section were all reported for the reference grain boundary cohesive strength $\Sigma_{\max }=0.5 \mathrm{GPa}$. Here, we investigate the sensitivity of the macroscopic toughness to hydrogen loading as a function of the grain boundary cohesive strength $\Sigma_{\max }$ in the slow diffusion limit.

In addition to the reference case of $\Sigma_{\max }=0.5 \mathrm{GPa}$, we consider a case of a high cohesive strength with $\Sigma_{\max }=1 \mathrm{GPa}$ and $\delta_{n}=1.5 b$, as well as a case of an intermediate cohesive strength with $\Sigma_{\max }=0.75 \mathrm{GPa}$ and $\delta_{n}=2 b$. The values of $\delta_{n}=1.5 b$ and $\delta_{n}=2 b$ are chosen so that in all cases $K_{0}$ remains unchanged from the reference case. Predictions of $K_{\mathrm{ss}} / K_{0}$ and the corresponding $\rho_{\mathrm{ss}}$ versus hydrogen loading parametrised by $\theta_{0}$ are plotted in Figs. $8(\mathrm{a})$ and $8(\mathrm{~b})$, respectively for $\Sigma_{\max }=0.75 \mathrm{GPa}$ and $\Sigma_{\max }=1 \mathrm{GPa}$ in addition to the reference value of $\Sigma_{\max }=0.5 \mathrm{GPa}$. While the sensitivity of the toughness to $\theta_{0}$ is rather similar in all three cases, the normalised toughness is higher in the $\Sigma_{\max }=1 \mathrm{GPa}$ case. This is due to the fact that the dislocation density at steady-state crack growth is higher for the higher cohesive strength case (see Fig. 8(b)) which in turn results in a higher dissipation and hence a larger normalised macroscopic toughness $K_{\mathrm{ss}} / K_{0}$.

In the slow diffusion limit, $\theta_{T}=\theta_{0}$ and thus the cohesive strength $\sigma_{\max }$ is directly related to $\theta_{0}$ via 


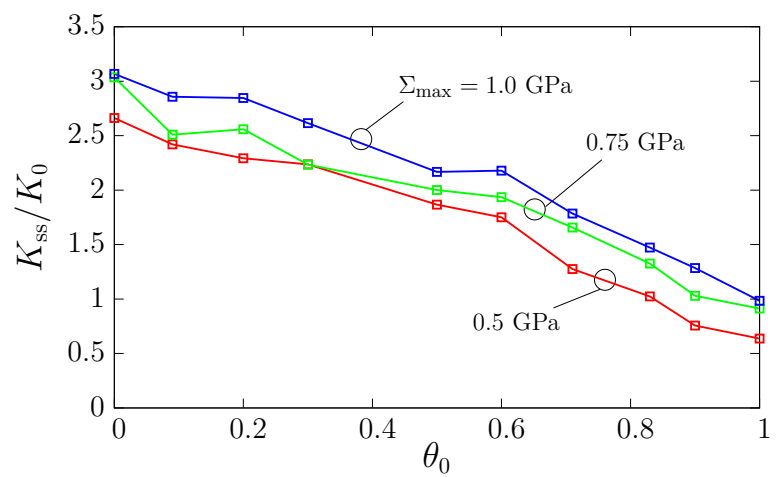

(a)

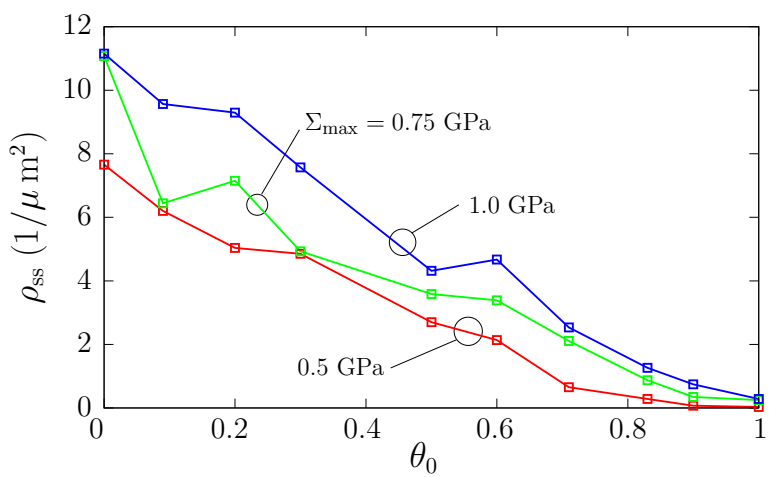

(b)

Figure 8: Predictions of the (a) normalised steady-state toughness $K_{\mathrm{ss}} / K_{0}$ and (b) steady-state dislocation density $\rho_{\mathrm{ss}}$ versus the hydrogen loading parametrised by $\theta_{0}$. Results are shown for the slow diffusion case and three values of the cohesive strength $\Sigma_{\max }$ with $K_{0}$ kept fixed at the reference value.

Eq. (15). Thus, the predictions of steady-state toughness $K_{\mathrm{ss}}$ can be summarised in terms of two nondimensional groups that characterise the fracture properties of the material in the presence of hydrogen, viz. $\sigma_{\max } / \sigma_{Y}$ and $\bar{\delta}_{n} \equiv \delta_{n} / b$. The DDP predictions of the normalised toughness $K_{\mathrm{ss}} /(G \sqrt{b})$ versus $\sigma_{\max } / \sigma_{Y}$ are included in Fig. $9^{3}$ for selected values of $\bar{\delta}_{n}$. It shall be emphasised that in the predictions shown in Fig. 9 the values of $\delta_{n}$ and $\sigma_{\max }$ are varied independently. Therefore, in Fig. 9, unlike the calculations of Fig. 8 the value of $K_{0}$ is not fixed. Recall that $\bar{\delta}_{n}$ and $\sigma_{Y}$ are not affected by hydrogen loading while $\sigma_{\max }$ varies with $\theta_{0}$ as per Eq. (15). Thus, each curve in Fig. 9 represents the sensitivity of the toughness to hydrogen loading for a given material with a unique value of $\bar{\delta}_{n}$. Moreover, we chose to normalise $K_{\mathrm{ss}}$ by $G \sqrt{b}$ as this parameter is independent of $\delta_{n}$ and $\theta_{0}$ and thus the $K_{\mathrm{ss}}$ values of the curves in Fig. 9 are directly comparable. Two clear features emerge from Fig. 9:

(i) At a fixed $\sigma_{\max } / \sigma_{Y}$, the toughness $K_{\mathrm{ss}} /(G \sqrt{b})$ is lower for curves with smaller $\bar{\delta}_{n}$.

(ii) The normalised toughness $K_{\mathrm{ss}} /(G \sqrt{b})$ increases with increasing $\sigma_{\max } / \sigma_{Y}$ and the rate of growth is lower for curves with smaller $\bar{\delta}_{n}$. This suggests that the toughness of materials with a lower $\bar{\delta}_{n}$ is more sensitive to a reduction in $\sigma_{\max } / \sigma_{Y}$, as for a given value of $\sigma_{\max } / \sigma_{Y}$, the magnitude of $K_{\mathrm{Ss}}$ is higher for curves with higher $\bar{\delta}_{n}$.

The results in Fig. 9 can be used to estimate the toughness of the material due to hydrogen loading as follows: The value of $\bar{\delta}_{n}$ is known independent of the hydrogen loading thus fixed for a given material. For a given hydrogen loading, Eq. (15) can be used to estimate the normalised cohesive strength $\sigma_{\max } / \sigma_{Y}$ and then the data in Fig. 9 directly gives the steady-state toughness $K_{\mathrm{ss}}$. An inference that can be drawn from the summary in Fig. 9 is that the toughness of materials with low values of $\bar{\delta}_{n}$ are expected to be more sensitive to hydrogen loading. This result might be used as a guide in grain boundary engineering to design alloys that are less sensitive to hydrogen loading.

\section{Discussion}

In the past years, a large number of hydrogen embrittlement theories have been proposed in order to rationalise the embrittlement of metals due to hydrogen; see recent review by Robertson et al. (2015).

\footnotetext{
${ }^{3}$ Some of the curves of Fig. 9 are incomplete as performing calculations with both high values of $\sigma_{\text {max }}$ and $\bar{\delta}_{n}$ is numerically prohibitive due to the large plastic zone sizes.
} 


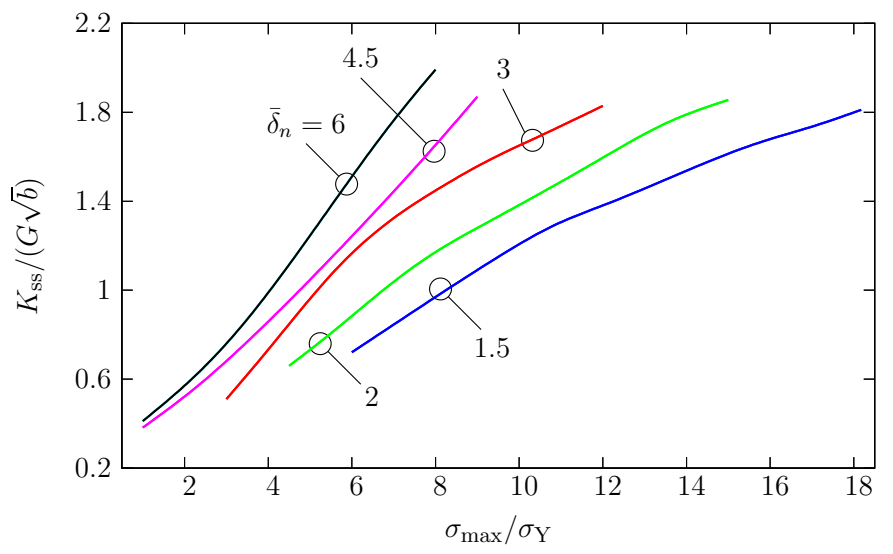

Figure 9: Summary of the DDP predictions of the normalised toughness $K_{\mathrm{ss}} /(G \sqrt{b})$ versus the normalised cohesive strength $\sigma_{\max } / \sigma_{Y}$ as in the presence of hydrogen. Results are included for materials with five selected values of the normalised cohesive opening $\bar{\delta}_{n}$.

While some of these mechanisms such as hydrogen enhanced dislocation ejection from free-surfaces or near surface regions (Lynch, 1979) are now considered questionable, the applicability of some other theories also remains uncertain. This is due to a lack of precise test data on hydrogen concentration and a lack of detailed quantitative comparisons between the measured and predicted mechanical strengths of a given material for a range of specimen geometries, states of absorbed hydrogen and types of heat treatment. Moreover, it is likely that a number of embrittlement mechanisms exist, with the dominant mechanism dependent upon microstructure, hydrogen concentration, temperature, and so on. Nevertheless, inconsistencies in the predictive ability of current models persist and here we will explore the deficiencies of not only the model developed here but also some related models in the literature.

A large literature suggests that hydrogen does not affect the overall tensile response of metals (e.g. steels) prior to fracture with the main effect of hydrogen being the reduction in the ductility and fracture strength; see for example Gangloff (2003). Models consistent with this hypothesis include the so-called HID mechanism as analysed here and a more recent model proposed by Song and Curtin (2013) which suggests that hydrogen embrittlement is closely linked to the prevention of dislocation emission from the crack tip (thereby suppressing crack tip blunting). In their work, Song and Curtin (2013) performed a molecular dynamics (MD) simulation of a crack tip, with an outer imposed $K$-field. In their simulations they only capture dislocation nucleations from the surface as there are no Frank-Read sources present in their model. Their results show that in the absence of hydrogen, crack tip dislocation emission blunts the crack tip and prevents crack growth. Yet, if sufficient hydrogen enters the material, accumulation of hydrogen at the crack tip allows for cleavage before dislocation emission. Taking this further Song and Curtin (2013) consider a range of steels with grain sizes on the order of $30 \mu \mathrm{m}$ and use their MD simulations to determine whether a flaw on the order of the grain size will lead to cleavage or crack tip blunting; see their Table 1. Their analysis shows remarkable agreement with experimental data on whether embrittlement is observed or not. However, an inconsistency emerges when the model is compared with independent fracture toughness measurements. For example, the growth of the $30 \mu \mathrm{m}$ flaw at approximately the yield strength implies that the toughness of the steel in the presence of hydrogen is on the order of $5 \mathrm{MPam}^{1 / 2}$. However the measured values of threshold toughness for such steels are typically in the range of $20-60 \mathrm{MPam}^{1 / 2}$. Conventional fracture mechanics then suggests that the transition flaw for a steel of toughness $20-60 \mathrm{MPa} \mathrm{m}^{1 / 2}$ and yield strength $\sigma_{Y}=500 \mathrm{MPa}$ is $a_{T}=\left(K_{\mathrm{IC}} / \sigma_{Y}\right)^{2} / \pi \approx 0.2 \mathrm{~mm}$, implying that a $30 \mu \mathrm{m}$ defect should have only a minor effect upon the strength. This insensitivity is not addressed by Song and Curtin (2013) and suggests that some additional physics is needed in order to understand cleavage in the presence of hydrogen.

The HID mechanism as analysed here assumes that the only effect of hydrogen is to reduce the cohesive strength $\sigma_{\max }$ (and consequently also the cohesive energy) and thereby promoting quasi-brittle fracture. 
Models based on this general idea have received extensive attention in the literature but a common thread is the requirement of a high hydrogen concentration at the crack tip. Crack tip analyses based on conventional plasticity restrict the hydrostatic tensile stresses at the crack tip in an ideally plastic solid to about $3 \sigma_{Y}$ : taking $\sigma_{Y}=300 \mathrm{MPa}$ and $V_{\mathrm{H}}=2 \times 10^{-6} \mathrm{~m}^{3} / \mathrm{mol}$, Eq. (12) predicts that the crack tip hydrogen concentration is only about twice the remote concentration at room temperature. This, has driven the application of the so-called higher order or gradient plasticity models which allow for the generation of higher crack tip stresses due to the presence of geometrically necessary dislocations near the crack tip. For example Martínez-Pañeda et al. (2016) recently used such a model in the context of the HID model of Gerberich (2012) to demonstrate that fracture by HID can occur due to high hydrogen concentrations at the crack tip resulting from high stresses. However, in that analysis Martínez-Pañeda et al. (2016) used a plasticity length scale of $\ell=5 \mu \mathrm{m}$ in order to generate the required large crack tip opening stresses. The choice of this large value of $\ell$ is misleading as independent measurements of $\ell$ (e.g. from fitting to indentation data) suggest that $\ell \leq 0.1 \mu \mathrm{m}$ : the use of $\ell=0.1 \mu \mathrm{m}$ would not give sufficiently high stresses to drive in significant amounts of hydrogen to the crack tip.

The current study builds on the HID hypothesis by using discrete dislocation plasticity wherein the dislocation structures and the associated crack tip stresses are generated as a natural outcome of the boundary value problem solution rather than being inputted as an arbitrary material parameter via a length scale. Our calculations suggest that the stresses generated around the crack tip are not sufficiently high in order to draw in significant levels of hydrogen resulting in the predictions of the R-curves being approximately similar in both the fast and slow diffusion cases. Instead, the high trap binding energies (i.e. large negative values of $\Delta H$ ) draw in hydrogen to grain boundaries independent of the crack tip stress fields, reducing the cohesive strength and resulting in a quasi-brittle fracture. Nevertheless, similar to all models proposed in the literature to-date, inconsistencies persist also with the analysis presented here. For example, similar to Song and Curtin (2013) the current analysis predicts that hydrogen loading will significantly reduce the fracture toughness of the metal. As discussed above, this is not supported by fracture toughness measurements which suggest only a mild sensitivity of fracture toughness to hydrogen loading but a large reduction in the tensile strength (or ductility) with increasing hydrogen concentration.

The analysis presented here suggests that HID mechanism, if operative, is not a result of hydrogen being drawn into the crack tip by the high stresses associated with the crack tip fields. Nevertheless, it is worth emphasising the limitations of the analysis presented here. For example, the toughness values reported here (even in the absence of hydrogen) are nearly an order of magnitude lower than the corresponding values for Al alloys. The discrepancy may be due to several idealisations in the model. For example, for numerical reasons, we have used a $\sigma_{\max }$ value that is about a factor of 5 smaller than the representative value for the bond strength of $\mathrm{Al}$ and the amount of plastic dissipation accompanying crack growth will increase with increasing cohesive strength; a high loading rate is used to reduce the computing time and this also acts to decrease the amount of plastic dissipation; the model is purely two dimensional, with both three-dimensional dislocation effects and three-dimensional crack growth effects neglected; and the effects of crack tip blunting are not taken into account in our small strain analyses. However, it is also possible that at least some of the discrepancy arises because of the fact that the calculations are carried out for small amounts of a straight crack growth in a single crystal, while experimental values typically pertain to much larger amounts of crack growth in a polycrystal, with effects such as crack growth off the initial crack plane and interactions with grain boundaries possibly coming into play.

\section{Concluding remarks}

We have presented the analysis of mode-I fracture of a single crystal with plastic flow in the crystal described by discrete dislocation plasticity (DDP) and material separation (decohesion) modelled using a cohesive zone formulation. The computations are motivated by the analysis of hydrogen induced decohesion (HID) along grain boundaries where hydrogen decreases the strength of grain boundaries thereby allowing a relatively 
easy propagation of cracks along these boundaries. The motion of dislocations was assumed to be unaffected by hydrogen (i.e. effects such as Hydrogen enhanced localised plasticity was neglected) but hydrogen was assumed to reduce the cohesive strength of the grain boundaries based on the local hydrogen concentration.

Dislocations play a dual role in the plastic flow. On the one hand, the motion of dislocations results in plastic flow and dissipation while dislocations also act as stress concentrators that raise the stress level in their vicinity. The hydrostatic tensile field created by individual dislocations attracts hydrogen and we have investigated two limiting cases to quantify this impact of dislocations. In the fast hydrogen diffusion limit we assumed that hydrogen was always maintained at chemical equilibrium within the material so that there was a high hydrogen concentration in the regions of high tensile hydrostatic stress. By contrast, in the slow diffusion limit we assumed that over the duration of the test hydrogen did not diffuse appreciably and therefore remained at its initial spatially uniform concentration. In both cases, the predicted toughness decreased with increasing initial hydrogen concentration. The lower cohesive strength at high hydrogen concentrations results in reduced dislocation activity around the crack tip and reduced material toughness. In fact, at the highest hydrogen concentrations analysed here, crack growth occurred primarily in an elastic manner. Surprisingly, the toughness in the fast diffusion limit was only about $12 \%$ lower compared to the slow diffusion limit and these differences were only present at intermediate hydrogen concentrations. This was due to the fact that the local tensile hydrostatic stresses generated by both the dislocation structures and the crack tip field locally increased the hydrogen concentration by not more than $40 \%$ over the hydrogen concentration of the stress-free material. This conclusion is contrary to what is typically assumed and included in models via the use of large plasticity length scales in gradient plasticity modelling of hydrogen embrittlement.

We conclude by developing a map that summarises the sensitivity of the steady-state toughness of the material to hydrogen concentration. In particular the DDP calculations predict that the toughness of materials with a small cohesive opening at the peak cohesive traction is more sensitive to hydrogen loading. We speculate that this result might be used as a guide in grain boundary engineering to design alloys that are less sensitive to hydrogen embrittlement by the HID mechanism.

\section{References}

Bechtlea, S., Kumar, M., Somerday, B. P., Launeye, M. E., Ritchie, R. O., 2013. Grain-boundary engineering markedly reduces susceptibility to intergranular hydrogen embrittlement in metallic materials. Acta Materialia 57, 4148-4157.

Bhandakkar, T. K., Chng, A. C., Curtin, W., Gao, H., 2010. Dislocation shielding of a cohesive crack. Journal of the Mechanics and Physics of Solids 58 (4), 530-541.

Birnbaum, H., Sofronis, P., 1994. Hydrogen-enhanced localized plasticity a mechanism for hydrogen-related fracture. Materials Science and Engineering: A 176, 191-202.

Chakravarthy, S. S., Curtin, W. A., 2011. New algorithms for discrete dislocation modeling of fracture. Modelling and Simulation in Materials Science and Engineering 19 (4), 045009.

Chen, J. Y., Wei, Y., Huang, Y., Hutchinson, J. W., Hwang, K. C., 1999. The crack tip fields in strain gradient plasticity: the asymptotic and numerical analysis. Engineering Fracture Mechanics 64, 625-648.

Cleveringa, H. H. M., Van der Giessen, E., Needleman, A., 2000. A discrete dislocation analysis of mode i crack growth. Journal of the Mechanics and Physics of Solids 48 (6), 1133-1157.

Cleveringa, H. H. M., Van der Giessen, E., Needleman, A., 2001. A discrete dislocation analysis of rate effects on mode i crack growth. Materials Science and Engineering A 317, 37-43.

Deshpande, V. S., Needleman, A., Van der Giessen, E., 2002. Discrete dislocation modeling of fatigue crack propagation. Acta Materialia 50, 831-846.

Gangloff, R., 2003. Hydrogen-assisted cracking. In: Milne, E., Ritchie, R. O., Karihaloo, B. (Eds.), Comprehensive Structural Integrity. Pergamon, Oxford, pp. 31-101.

Geng, W. T., Freeman, A. J., Olson, G. B., Tateyama, Y., Ohno, T., 2005. Hydrogen-promoted grain boundary embrittlement and vacancy activity in metals: Insights from Ab initio total energy calculatons. Materials Transactions 46, 756-760.

Gerberich, W., 2012. 8 - modeling hydrogen induced damage mechanisms in metals. In: Gangloff, R. P., Somerday, B. P. (Eds.), Gaseous Hydrogen Embrittlement of Materials in Energy Technologies. Vol. 1 of Woodhead Publishing Series in Metals and Surface Engineering. Woodhead Publishing, pp. $209-246$.

Jiang, D., Carter, E. A., 2004. First principles assessment of ideal fracture energies of materials with mobile impurities: implications for hydrogen embrittlement of metals. Acta Materialia 52, 4801-4807.

Kubin, L. P., Canova, G., Condat, M., Devincre, B., Pontikis, V. Brechet, Y., 1992. Dislocation microstructures and plastic flow: a 3d simulation. Solid State Phenomena 23-24, 455-472. 
Lynch, S., 1979. Hydrogen embrittlement and liquid-metal embrittlement in nickel single crystals. Scripta Metallurgica 13 (11), $1051-1056$

Martínez-Pañeda, E., Niordson, C. F., Gangloff, R. P., 2016. Strain gradient plasticity-based modeling of hydrogen environment assisted cracking. Acta Materialia 117, 321-332.

Novak, P., Yuan, R., Somerday, B. P., Sofronis, P., Ritchie, R. O., 2010. A statistical, physical-based, micro-mechanical model of hydrogen-induced intergranular fracture in steel. Journal of the Mechanics and Physics of Solids 58 (2), 206-226.

Oriani, R., 1970. The diffusion and trapping of hydrogen in steel. Acta Metallurgica 18, 147-157.

Rice, J. R., 1987. Tensile crack tip fields in elastic-ideally plastic crystals. Mechanics of Materials 6, $317-335$.

Robertson, I., Sofronis, P., Nagao, A., Martin, M., Wang, S., Gross, D., Nygren, K., 6 2015. Hydrogen embrittlement understood. Metallurgical and Materials Transactions B: Process Metallurgy and Materials Processing Science 46 (3), $1085-1103$.

Serebrinsky, S., Carter, E. A., Ortiz, M., 2004. A quantum-mechanically informed continuum model of hydrogen embrittlement. Journal of the Mechanics and Physics of Solids 52 (10), 2403-2430.

Sofronis, P., McMeeking, R. M., 1989. Numerical analysis of hydrogen transport near a blunting crack tip. Journal of the Mechanics and Physics of Solids 37 (3), 317-350.

Song, J., Curtin, W. A., 2013. Atomic mechanism and prediction of hydrogen embrittlement in iron. Nature materials 12 (2), $145-151$

Suo, Z., Shih, C. F., Varias, A. G., 1993. A theory for cleavage cracking in the presence of plastic flow. Acta Metallurgica et Materialia 41, 1551-1557.

Troiano, A. R., 1960. The role of hydrogen and other interstitials in the mechanical behaviour of metals. Trans. ASM. 52, 54-80.

Tvergaard, V., Hutchinson, J. W., 1992. The relation between crack growth resistance and fracture process parameters in elastic-plastic solids. Journal of the Mechanics and Physics of Solids 40 (6), 1377-1397.

Van der Giessen, E., Needleman, A., 1995. Discrete dislocation plasticity: a simple planar model. Modelling and Simulation in Materials Science and Engineering 3, 689-735.

Van der Ven, A., Ceder, G., 2003. Impurity-induced van der waals transition during decohesion. Physical Review B 67 (6), 060101-1-4

\section{Appendix A. Hydrogen trapped in dislocation structures}

As discussed in the main body of the paper dislocations play a dual role in plastic flow. On one hand the motion of dislocations results in plastic deformation and dissipation and on the other hand dislocations act as local stress concentrators. The tensile hydrostatic stress associated with the fields of these dislocations can attract hydrogen and increase the concentration of hydrogen within certain types of dislocation structures. Dislocation structures with a large net Burgers vector (i.e. geometrically necessary dislocation structures) will have high stress fields and thus are most likely to trap hydrogen. Here, we analyse the hydrogen trapped in one such extreme dislocation structure comprising an infinite cubic array of positive edge dislocations with Burgers vector $b$ as sketched in Fig. A.1. The dislocations are assumed to be spaced at a distance $h$ in both the $x$ - and $y$-directions so that the dislocation density within this array is $\rho_{\text {dis }}=1 / h^{2}$. Since the dislocation array is periodic it suffices to analyse a single unit cell as sketched in Fig. A.1. This infinite dislocation array is assumed to be in a material within a hydrogen environment that maintains the hydrogen concentration at $C_{L}^{0}$ within the stress-free material (i.e. in the absence of the dislocation array) at equilibrium. We then introduce the dislocation array and again let hydrogen attain chemical equilibrium. Using Eq. (12) the average hydrogen concentration within this unit cell (and hence within the array) is given as

$$
\bar{C}=\frac{1}{h^{2}-\pi r_{c}^{2}} \int_{\Omega / \Omega_{\mathrm{c}}} C\left(x_{i}\right) d \Omega=\frac{C_{L}^{0}}{h^{2}-\pi r_{c}^{2}} \int_{\Omega / \Omega_{\mathrm{c}}} \exp \left(\frac{\sigma_{h}\left(x_{i}\right) V_{\mathrm{H}}}{R T}\right) d \Omega,
$$

where $\Omega=h^{2}$ is the area of the unit cell and $\Omega_{c}=\pi r_{c}^{2}$ is the area of the dislocation core taken here to be a circle of radius $r_{c}$ centred at the position of the edge dislocation. The hydrostatic stress field $\sigma_{h} \equiv \sigma_{k k} / 3$ 
for this infinite array of dislocations is given by (see Appendix B for details of the derivation)

$$
\begin{aligned}
\sigma_{k k}\left(\xi_{n}, \eta_{n}\right)=-\frac{2 k_{0} b}{h} \pi(1+\nu) & \left\{\frac{\sinh \left(2 \pi \eta_{n}\right)}{\cosh \left(2 \pi \eta_{n}\right)-\cos \left(2 \pi \xi_{n}\right)}+\right. \\
& \left.\sum_{m=1}^{+\infty} \frac{2 \sinh \left(2 \pi \eta_{n}\right)\left[\cosh \left(2 \pi \eta_{n}\right)-\cos \left(2 \pi \xi_{n}\right) \cosh (2 \pi m)\right]}{\left[\cosh \left(2 \pi \eta_{n}\right) \cosh (2 \pi m)-\cos \left(2 \pi \xi_{n}\right)\right]^{2}-\left[\sinh \left(2 \pi \eta_{n}\right) \sinh (2 \pi m)\right]^{2}}\right\} .
\end{aligned}
$$

where $k_{0}=G / 2 \pi(1-\nu)$ and

$$
\xi_{n}=\frac{x_{n}}{h}, \quad \eta_{n}=\frac{y_{n}}{h} .
$$

Predictions of the normalised average hydrogen concentration $\bar{C} / C_{L}^{0}$ trapped in the lattice by the dislocation

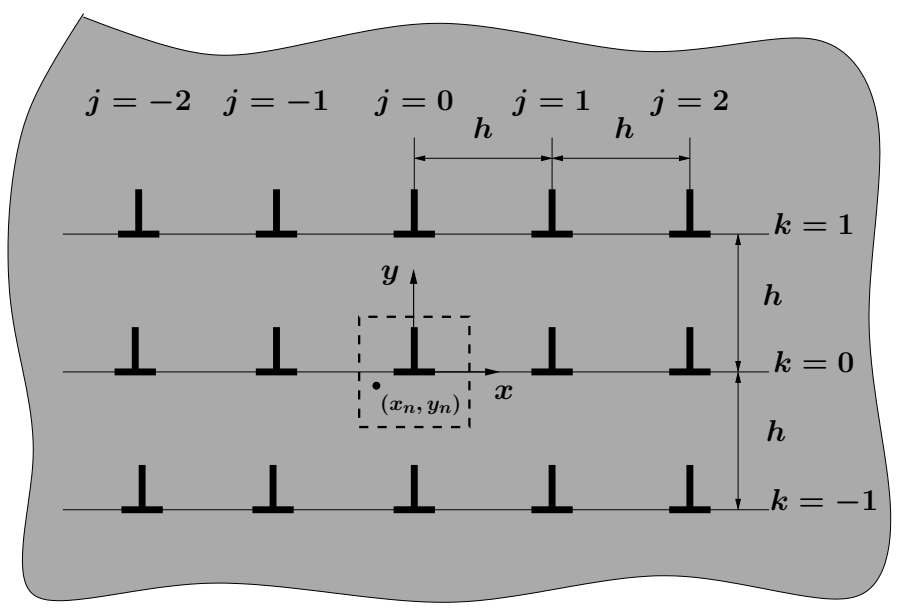

Figure A.1: Sketch of the doubly periodic infinite cubic array of positive edge dislocations spaced a distance $h$ apart. The coordinate system used as well as the unit cell employed in the analysis are marked.

stress field is plotted in Fig. A.2 as a function of the dislocation density $\rho_{\text {dis }}$ at a temperature $T=300 \mathrm{~K}$ and representative values for material properties of aluminium, i.e. $E=70 \mathrm{GPa}, \nu=0.33, b=0.25 \mathrm{~nm}$ and $V_{\mathrm{H}}=2 \times 10^{-6} \mathrm{~m}^{3} / \mathrm{mol}$. Results are shown for three choices of the core radius $r_{c}=1.25 b, 2.5 b$ and $5 b$ for dislocation densities up to a relatively high value of $1.2 \times 10^{16} \mathrm{~m}^{-2}$. Even at such high dislocation density with a core radius of just $1.25 b$, the average concentration of hydrogen trapped in the lattice by the stress field is enhanced by less than $10 \%$ compared to $C_{L}^{0}$. We thus conclude that the stress concentrations due to the dislocations do not have a significant effect on the equilibrium hydrogen distribution within the lattice.

We emphasise here that this analysis neglects the hydrogen trapped within the dislocation cores. However, the hydrogen within the dislocation cores is unlikely to be available to interact with grain boundaries and thus it is not expected to contribute to the HID mechanism analysed here.

\section{Appendix B. Derivation of hydrostatic stress field due to an infi- nite cubic array of positive edge dislocations}

Consider an infinite cubic array of positive edge dislocations with Burgers vector $b$ spaced at distance $h$ apart in both the $x$ - and $y$-directions as sketched in Fig. A.1. With one of these edge dislocations located at the origin of the coordinate system the location of dislocation $(j, k)$ is then given as $(j h, k h)$ and the distance of material point $\left(x_{n}, y_{n}\right)$ from a dislocation $(j, k)$ in the $x$ - and $y$-directions is

$$
\Delta x=x_{n}-j h, \quad \text { and } \quad \Delta y=y_{n}-k h \quad(j, k=\ldots,-1,0,1, \ldots),
$$




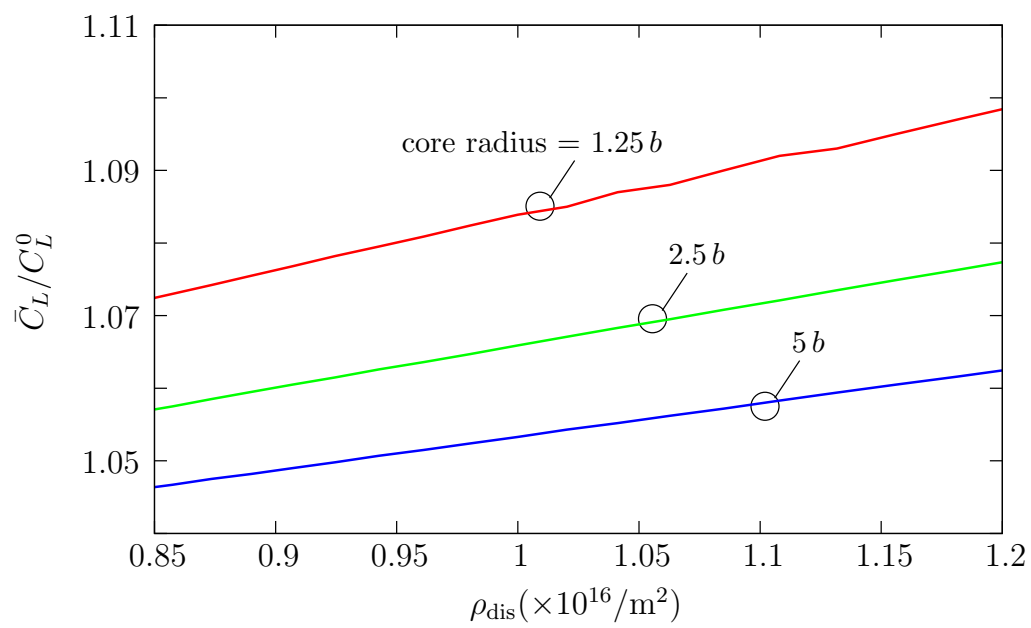

Figure A.2: Predictions of the normalised average concentration $\bar{C} / C_{L}^{0}$ of the hydrogen trapped in the lattice by the dislocation stress fields as a function of the dislocation array density $\rho_{\text {dis }} \equiv 1 / h^{2}$. Results are shown for three values of the assumed core radius $r_{c}$.

respectively. Using the plane strain infinite-body stress fields of edge dislocations in an infinite medium, the direct stress components in the $x$ - and $y$-directions at material point $\left(x_{n}, y_{n}\right)$ follow as

$$
\begin{aligned}
\sigma_{x x} & =-\frac{k_{0} b}{h} \sum_{k=-\infty}^{+\infty} \sum_{j=-\infty}^{+\infty} \frac{\left(\eta_{n}-k\right)\left[3\left(\xi_{n}-j\right)^{2}+\left(\eta_{n}-k\right)^{2}\right]}{\left[\left(\xi_{n}-j\right)^{2}+\left(\eta_{n}-k\right)^{2}\right]^{2}}, \\
\sigma_{y y} & =+\frac{k_{0} b}{h} \sum_{k=-\infty}^{+\infty} \sum_{j=-\infty}^{+\infty} \frac{\left(\eta_{n}-k\right)\left[\left(\xi_{n}-j\right)^{2}+\left(\eta_{n}-k\right)^{2}\right]}{\left[\left(\xi_{n}-j\right)^{2}+\left(\eta_{n}-k\right)^{2}\right]^{2}}
\end{aligned}
$$

where $k_{0}=G / 2 \pi(1-\nu)$ and

$$
\xi_{n}=\frac{x_{n}}{h} \quad \text { and } \quad \eta_{n}=\frac{y_{n}}{h} .
$$

We wish to determine the hydrostatic stress $\sigma_{h}=\sigma_{k k} / 3$. Linear isotropic Hooke's law dictates that under plane strain conditions

$$
\sigma_{k k}=\sigma_{x x}+\sigma_{y y}+\sigma_{z z}=(1+\nu)\left(\sigma_{x x}+\sigma_{y y}\right),
$$

and thus the problem reduces to determining $\sigma_{x x}+\sigma_{y y}$. Using the ideas introduced by Van der Giessen and Needleman (1995) for periodic strings of dislocations in the $x$-direction, Eq. (B.2) simplifies to

$$
\begin{gathered}
\sigma_{x x}=-\frac{k_{0} b}{h} \pi \sum_{k=-\infty}^{+\infty} \frac{1}{\cosh \left(2 \pi\left(\eta_{n}-k\right)\right)-\cos \left(2 \pi \xi_{n}\right)}\left\{2 \sinh \left(2 \pi\left(\eta_{n}-k\right)\right)+\right. \\
\left.2 \pi\left(\eta_{n}-k\right) \frac{1-\cosh \left(2 \pi\left(\eta_{n}-k\right)\right) \cos \left(2 \pi \xi_{n}\right)}{\cosh \left(2 \pi\left(\eta_{n}-k\right)\right)-\cos \left(2 \pi \xi_{n}\right)}\right\}, \\
\sigma_{y y}=+\frac{k_{0} b}{h} \pi \sum_{k=-\infty}^{+\infty} 2 \pi\left(\eta_{n}-k\right) \frac{1-\cosh \left(2 \pi\left(\eta_{n}-k\right)\right) \cos \left(2 \pi \xi_{n}\right)}{\left[\cosh \left(2 \pi\left(\eta_{n}-k\right)\right)-\cos \left(2 \pi \xi_{n}\right)\right]^{2}},
\end{gathered}
$$

and therefore,

$$
\sigma_{x x}+\sigma_{y y}=-\frac{2 k_{0} b}{h} \pi \sum_{k=-\infty}^{+\infty} \frac{\sinh \left(2 \pi\left(\eta_{n}-k\right)\right)}{\cosh \left(2 \pi\left(\eta_{n}-k\right)\right)-\cos \left(2 \pi \xi_{n}\right)}
$$


Finally,

$$
\sigma_{k k}\left(\xi_{n}, \eta_{n}\right)=-\frac{2 k_{0} b}{h} \pi(1+\nu) \sum_{k=-\infty}^{+\infty} \frac{\sinh \left(2 \pi\left(\eta_{n}-k\right)\right)}{\cosh \left(2 \pi\left(\eta_{n}-k\right)\right)-\cos \left(2 \pi \xi_{n}\right)},
$$

where further elaboration of Eq. (B.6) gives

$$
\begin{aligned}
\sigma_{k k}\left(\xi_{n}, \eta_{n}\right)=-\frac{2 k_{0} b}{h} \pi(1+\nu) & \left\{\frac{\sinh \left(2 \pi \eta_{n}\right)}{\cosh \left(2 \pi \eta_{n}\right)-\cos \left(2 \pi \xi_{n}\right)}+\right. \\
& \left.\sum_{m=1}^{+\infty} \frac{2 \sinh \left(2 \pi \eta_{n}\right)\left[\cosh \left(2 \pi \eta_{n}\right)-\cos \left(2 \pi \xi_{n}\right) \cosh (2 \pi m)\right]}{\left[\cosh \left(2 \pi \eta_{n}\right) \cosh (2 \pi m)-\cos \left(2 \pi \xi_{n}\right)\right]^{2}-\left[\sinh \left(2 \pi \eta_{n}\right) \sinh (2 \pi m)\right]^{2}}\right\} .
\end{aligned}
$$

\section{Appendix C. The variation in the results between the different realisations}

The results presented in this work are averages over five realisations of the source and obstacles distributions. To illustrate the variation in the results between the different realisations we re-plot the results of Fig. 6 in Fig. C.1 showing error bars that illustrate the maximum and minimum values obtained in the simulations of the 5 realisations. The variations in $K_{\mathrm{sS}} / K_{0}$ are relatively small while $\rho_{\mathrm{ss}}$ varies by larger amounts between the different realisations.

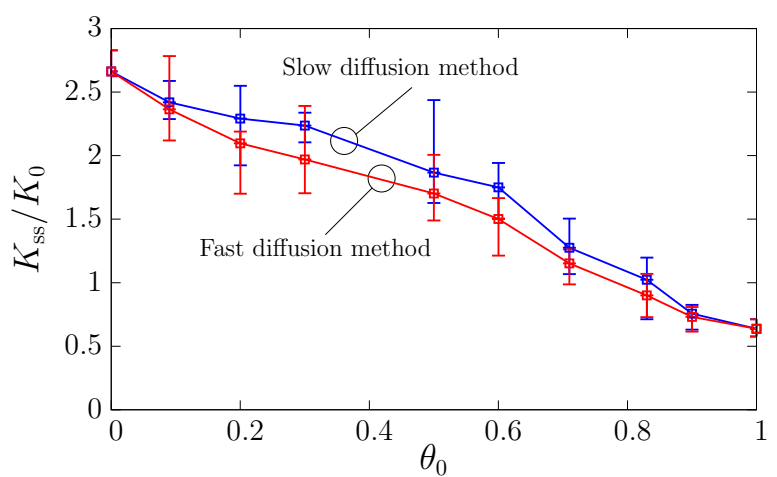

(a)

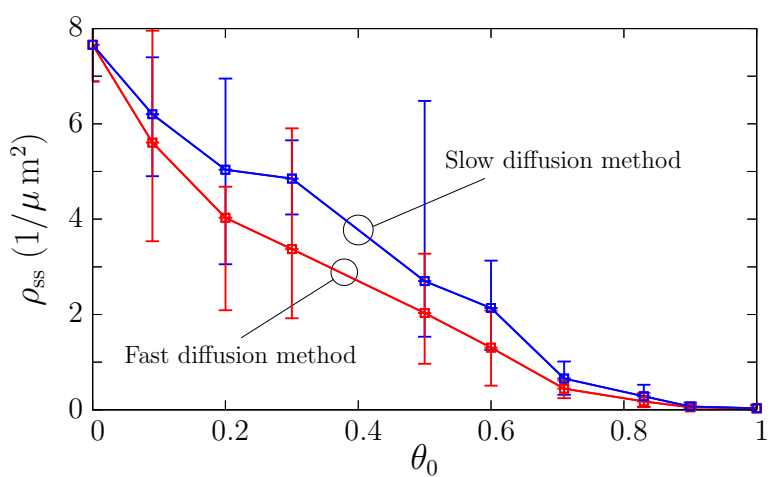

(b)

Figure C.1: Predictions of the (a) normalised steady-state toughness $K_{\mathrm{ss}} / K_{0}$ and (b) steady-state dislocation density $\rho_{\mathrm{ss}}$ as a function of the hydrogen loading parametrised by $\theta_{0}$. Results are shown for the slow and fast diffusion cases for the reference cohesive strength of $\Sigma_{\max }=0.5 \mathrm{GPa}$. The error bars indicate the variation in the results over the 5 realisations of the source and obstacle distributions considered here. 